\title{
Joint window and filter optimization for new waveforms in multicarrier systems

\author{
Ming-Fu Tang ${ }^{1 *}$ (D) and Borching Su ${ }^{1,2}$
}

\begin{abstract}
One of the demands of the next-generation wireless communication systems is being supportive to asynchronous traffic types. In order to meet this demand, many waveform candidates for next-generation wireless communication systems have low out-of-subband emissions (OOSBE) of the transmitted signal, and the popular ones deploy either filtering or windowing at the transmitter. In this paper, a joint windowing and filtering multi-carrier waveform based on a generalized orthogonal frequency division multiplexing (OFDM) system is proposed. A joint window and filter optimization problem to minimize OOSBE is also formulated. The optimization problem is first divided into two solvable subproblems using interior-point methods, and then an iterative algorithm is proposed to obtain the optimal window and filter pair. Simulation results suggest that the proposed joint windowing and filtering waveform has an advantage in suppressing OOSBE or enhancing spectral efficiency, and is more robust to frequency asynchronism compared to waveforms deploying only either filtering or windowing.
\end{abstract}

Keywords: Filtering-based waveform, Windowing-based waveform, Alternative optimization, Joint window and filter optimization, Multi-carrier modulation, Out-of-subband emissions

\section{Introduction}

New transmission waveforms beyond orthogonal frequency division multiplexing (OFDM) have been studied to a great extent recently, in order to meet the demands of next-generation communication systems [1]. Many new waveform proposals aim at suppressing sidelobe power or out-of-subband emissions (OOSBE) in generalized OFDM systems, since waveforms with this property may provide advantages such as low interference among asynchronous users accessing adjacent frequencies, spectral efficiency increase through reuse of guard frequencies, and enabling multi-service systems using mixed numerologies [2-7].

Among the many waveform proposals, filter bank multicarrier (FBMC) systems [8] possess perhaps the best sidelobe suppression performance when their associated pulse-shaping filters are properly designed. However, filters with this great property usually have a long impulse response, typically in the order of several symbol durations. This causes severe inter-symbol interference (ISI)

\footnotetext{
*Correspondence: d01942013@ntu.edu.tw

1 Graduate Institute of Communication Engineering, National Taiwan

University, No.1, Sec. 4, Roosevelt Road, Taipei 10617, Taiwan

Full list of author information is available at the end of the article
}

and results in difficulties in receiver designs especially when the signal is transmitted over multi-path channels [9]. With a similar transmitter structure, a windowingbased waveform, commonly known as weighted overlapand-add (WOLA) [4], has been proposed to suppress sidelobe powers without using long pulse shaping. Nevertheless, an extended guard interval, such as a cyclic prefix $(\mathrm{CP})$, is required for eliminating ISI caused by multi-path channels and the window of WOLA [10, 11], which leads to reduced spectral efficiency. In [12, 13], windowing schemes that require no guard interval extension were proposed to suppress sidelobe powers. However, the receiver structure in [12] causes signal-to-noise ratio (SNR) loss and leads to a degraded data reception performance, whereas the receiver structure in [13] leads to inter-carrier interference (ICI) between subcarriers for arbitrary window coefficients with multi-path fading channels.

In [14-16], precoding-based waveforms were proposed to suppress OOSBE by designing a precoder for transmitting data symbols. Generalized frequency division multiplexing (GFDM) [14] is able to suppress sidelobe power by using a circularly pulse-shaped precoder. In general, the GFDM precoder is represented by a non-unitary 
matrix[17]. The non-unitariness of such precoding matrix could lead to noise enhancement at the receiver, and thus, it degrades the performance of data reception. Spectrally precoded OFDM [15] and a power leakage-suppressing precoder [16] both possess unitary precoding matrices so that there is no noise enhancement at the receiver. However, the precoding-based waveforms require a number of null subcarriers, called virtual carriers (VCs), to be inserted in each subband, which will degrade spectral efficiency.

Recently, some new filtering-based waveforms were proposed with the objective to shorten the impulse response of the pulse shaping filters while maintaining a similar level of OOSBE suppression [18-25]. These waveforms take advantage of per-subband filtering to obtain a much shorter filter than FBMC, which executes persubcarrier filtering. Nevertheless, all filtering-based waveforms increase the delay spread of the equivalent channel and inevitably suffer from either a decreased spectral efficiency due to guard interval extension [21] or a receiver with a larger complexity for equalization of ISI effects. To trade a better OOSBE suppression performance, inserting a small number of VCs between adjacent subbands as additional guard bands is also considered [2, 22-24]. However, such insertion also leads to decreased spectral efficiency.

In this paper, a new waveform that jointly utilizes windowing in [12] and filtering at the transmitter is proposed. To suppress OOSBE, a subband-based joint window and filter optimization method is proposed. The proposed waveform using the proposed optimization method has an advantage in OOSBE suppression in contrast with previous filtering-based and windowing-based waveforms. Alternatively, the proposed waveform is more spectrally efficient for suppressing OOSBE to a specific level because it requires less VCs. Simulation results confirm the above advantages of the proposed waveform.

\subsection{Related waveforms}

The waveforms related to this research, including the aforementioned windowing-based and filtering-based waveforms, are further presented in the following.

Windowing-based waveforms: WOLA deploys nonmemoryless windowing at either the transmitter or the receiver [4]. To mitigate OOSBE, WOLA performed at the transmitter is introduced. A Nyquist window (e.g., raised-cosine) is usually adopted by current WOLA systems. However, an extended CP is required for ISI elimination. A waveform using memoryless windowing at the transmitter was proposed in [12]. This waveform has low OOSBE compared to the conventional OFDM and needs no extra CP. The objective of optimizing the window coefficients in [12] is to minimize the power leakage out of a subcarrier. Nevertheless, the receiver that assures orthogonality between subcarriers induces SNR loss caused by noise enhancement. A memoryless windowing-based waveform was also proposed in [13], which adopted a different receiver structure from that in [12]. Specifically, it gives up on assuring orthogonality between subcarriers for multi-path fading channels; thus, it results in ICI and endurable ISI.

Filtering-based waveforms: Universal-filtered multicarrier (UFMC) [18-20] was first proposed with the idea of using per-subband filtering to suppress OOSBE. Such subband-based filtering incurs mitigated ISI compared to FBMC. A UFMC system with an extended CP was proposed in [21] to further eliminate the ISI. With the similar idea of UFMC, filtered-OFDM (f-OFDM) [22, 23] considers a longer filter (up to a half symbol duration) to trade off even better OOSBE suppression at the expense of some manageable ISI. A UFMC system that employs WOLAbased windowing and specifically targets at low-latency applications has been proposed in [25].

\subsection{Contributions}

The major contributions of this paper are as follows:

- A per-subband-based waveform that exploits both windowing and filtering operations at the transmitter is proposed. This waveform is suitable for performing joint processing because there are two sets of design parameters. Unlike [25] which utilizes WOLA-based windowing, the windowing structure used in this paper is inspired by that in [12], which helps the CP length to be further reduced in contrast with the previous filtering-based waveforms. The proposed waveform exploits a subband-based filtering and windowing design, whereas a per-subcarrier design is executed in [12]. This design makes the proposed waveform more suitable for multi-subband systems (e.g., in [2]).

- A joint window and filter optimization method is proposed to suppress OOSBE of the proposed waveform. The proposed method enables the proposed waveform to possess advantages in spectral efficiency and OOSBE suppression over the previous filtering-based and windowing-based waveforms. The proposed method also facilitates the proposed waveform to induce much less SNR loss than the windowing-based waveform in [12] and to allow the use of a shorter CP for ISI elimination compared to WOLA and the previous filtering-based waveform to suppress OOSBE to a specific level.

\subsection{Organization and notations}

The remainder of this paper is organized as follows. Section 2 presents the system model. Section 3 addresses the joint window and filter design problem. The proposed 
optimization method is then described in Section 4. Simulation results are provided in Section 5, and Section 6 offers a conclusion.

Notations: Boldface lowercase letters represent column vectors; boldface uppercase letters represent matrices. The superscripts $(\cdot)^{*},(\cdot)^{T}$, and $(\cdot)^{H}$ denote the conjugate, transpose, and transpose-conjugate operations, respectively. The expected value operation is denoted as $E\{\cdot\}$. The vector $\mathbf{d}(\boldsymbol{\theta})$ of an $M$-length vector $\boldsymbol{\theta} \triangleq\left[\theta_{1} \cdots \theta_{M}\right]^{T}$

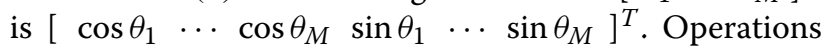
$\|\cdot\|$ and $\operatorname{diag}(\cdot) \operatorname{denote}$ the vector norm and vector/matrix diagonalization, respectively. Hadamard and Kronecker products between vectors $\mathbf{v}_{1}, \mathbf{v}_{2}$ are denoted as $\mathbf{v}_{1} \circ \mathbf{v}_{2}$ and $\mathbf{v}_{1} \otimes \mathbf{v}_{2}$, respectively. An identity matrix of size $M$ is denoted as $\mathbf{I}_{M}$; a zero matrix of size $M \times N$ is denoted as $\mathbf{0}_{M \times N}$. An $N$-length vector with all of its entries be equal to one is denoted as $\mathbf{1}_{N}$; a zero column vector of size $M$ is denoted as $\mathbf{0}_{M}$. A column vector $\mathbf{v}_{L}(z)$ of length $L$ is written as $\mathbf{v}_{L}(z)=\left[1 z \cdots z^{L-1}\right]^{T}$. An $M \times M$ circulant matrix $\mathbf{C}$ whose first column is $\left[c_{0} c_{1} \cdots c_{M-1}\right]^{T}$ is written as

$$
\mathbf{C}=\left[\begin{array}{cccc}
c_{0} & c_{M-1} & \cdots & c_{1} \\
c_{1} & c_{0} & \cdots & c_{2} \\
\vdots & \vdots & \ddots & \vdots \\
c_{M-1} & c_{M-2} & \cdots & c_{0}
\end{array}\right]
$$

A normalized discrete Fourier transform (DFT) matrix of size $M$ is $\mathbf{W}_{M}$. The $(m, n)$ th entry of the DFT matrix is $\left[\mathbf{W}_{M}\right]_{m, n}=\frac{1}{\sqrt{M}} e^{-j \frac{2 \pi}{M}(m-1)(n-1)}$. The domain of function $f$ is denoted as $\operatorname{dom} f$. The sets of non-negative and positive vectors of size $M$ are denoted as $\mathrm{R}_{+}^{M}$ and $\mathrm{R}_{++}^{M}$, respectively.

\section{System model}

\subsection{Transmitter structure}

Consider a multi-carrier system with $M$ subcarriers. Suppose that a communication device is allocated to a subband consisting of $P$ adjacent subcarriers. Figure 1 depicts the block diagram of the transmitter. Let $\mathbf{s}_{P}[n]$ denote the transmit symbol vector and assume

$$
\mathrm{E}\left\{\mathbf{s}_{P}[n] \mathbf{s}_{P}^{H}[m]\right\}=\left\{\begin{array}{l}
E_{S} \mathbf{I}_{P}, \quad n=m \\
\mathbf{0}_{P \times P}, \text { else }
\end{array}\right.
$$

where $E_{s}$ denotes the symbol energy. Now, the transmit symbol vector is precoded by an IDFT matrix $\mathbf{W}_{M}^{H}$, resulting in a vector $\mathbf{u}_{M}[n]=\mathbf{W}_{M}^{H} \mathbf{s}[n]$ where $\mathbf{s}[n]=$ $\left[\begin{array}{lll}\mathbf{0} & \mathbf{s}_{P}^{T}[n] & \mathbf{0}\end{array}\right]^{T}$.

To eliminate ISI and suppress the OOSBE, the vector $\mathbf{u}[n]$ is obtained by adding a CP of length $L$ to $\mathbf{u}_{M}[n]$ and multiplying a matrix consisting of window coefficients. Specifically, the windowed signal vector is expressed as $\mathbf{u}[n]=\operatorname{diag}\left(\left[w_{-L} \cdots w_{0} \cdots w_{M-1}\right]\right) \mathbf{R} \mathbf{u}_{M}[n]$, where $w_{m}, m=-L, \cdots, M-1$ are the window coefficients, and matrix $\mathbf{R}$, which represents adding the $\mathrm{CP}$, is written as

$$
\mathbf{R}=\left[\begin{array}{cc}
\mathbf{0}_{L \times(M-L)} & \mathbf{I}_{L} \\
\mathbf{I}_{M} &
\end{array}\right]
$$

Now, define

$$
W\left(e^{j \omega}\right)=\sum_{m=-L}^{M-1} w_{m} e^{-j(m+L) \omega}
$$

as the Fourier transform of the window coefficients, then the power spectral density (PSD) of signal $u[n]$, after parallel-to-serial $(\mathrm{P} / \mathrm{S})$ conversion of vector $\mathbf{u}[n]$, is [26]

$$
\mathcal{S}_{u}\left(e^{j \omega}\right)=\frac{E_{s}}{N} \sum_{k \in \mathcal{K}}\left|W\left(e^{j\left(\omega-\Omega_{k}\right)}\right)\right|^{2}
$$

where $N=M+L, \Omega_{k}=\frac{2 \pi k}{M}$ and $\mathcal{K}$ denotes the set of subcarriers that are actually used in a subband. Let $K$ denote the index of the subcarrier at the left of the allocated subband, then

$$
\mathcal{K} \triangleq\{K, K+1, \cdots, K+P-1\} .
$$

In conventional OFDM systems, the window coefficients are usually chosen as $w_{m}=1, \forall m=-L, \cdots, M-1$ referred to as a rectangular window. In this paper, the

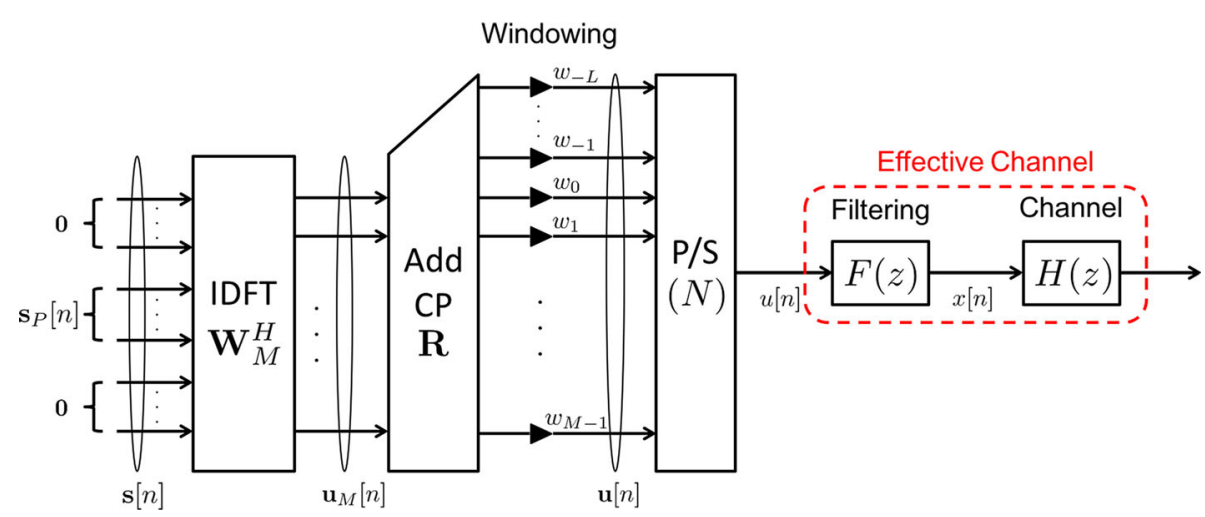

Fig. 1 Block diagram of the transmitter 
coefficients are considered to be more general, with a constraint that the power of the signal using any arbitrary coefficients is equal to that using the rectangular window. That is,

$$
\sum_{m=-L}^{M-1}\left|w_{m}\right|^{2}=N
$$

Note that the slope of sidelobe power decay of conventional OFDM signals is approximately proportional to the frequency distance to the center of the mainlobe [27]. The slow decay of sidelobe power in conventional OFDM systems results in large OOSBE.

An effective way of suppressing OOSBE is deploying per-subband filtering at the transmitter, which was first proposed in [18]. Subsequently, the windowed signal $u[n]$ is filtered by an FIR filter $F(z)$, which is defined as

$$
F(z)=\sum_{n=0}^{L_{f}-1} f[n] z^{-n}=\mathbf{v}_{L_{f}}^{T}\left(z^{-1}\right) \mathbf{f}
$$

where $f[n]$ is the impulse response and $L_{f}$ denotes the filter length, which is assumed to be much smaller than the number of subcarriers, i.e., $L_{f} \ll M$, and $\mathbf{f}=$ $\left[f[0] f[1] \cdots f\left[L_{f}-1\right]\right]^{T}$. Recall that the definition of $\mathbf{v}_{L_{f}}(z)$ is given in Section 1.3. Then, the PSD of the transmitted signal $x[n]$ is written as

$$
\mathcal{S}_{x}\left(e^{j \omega}\right)=\mathcal{S}_{u}\left(e^{j \omega}\right)\left|F\left(e^{j \omega}\right)\right|^{2} .
$$

The filter function $f[n], n=0, \cdots, L_{f}-1$, is chosen such that the expected value of the energy of the filtered signal $x[n]$ is equivalent to that of the windowed signal $u[n]$. Based on Parseval's relation and (3),

$$
\int_{-\pi}^{\pi} \mathcal{S}_{x}\left(e^{j \omega}\right) \frac{\mathrm{d} \omega}{2 \pi}=\int_{-\pi}^{\pi} \mathcal{S}_{u}\left(e^{j \omega}\right) \frac{\mathrm{d} \omega}{2 \pi}=P E_{s} .
$$

\subsection{Effective channel}

As illustrated in Fig. 1, the filtered signal $x[n]$ is sent over the channel $H(z)$. It is assumed that $H(z)$ is an FIR channel with a maximum order $L_{h}$, i.e.,

$$
H(z)=\sum_{n=0}^{L_{h}} h[n] z^{-n}
$$

where $h[n]$ denotes the impulse response of the FIR channel. By assuming that the transmitter and the receiver are perfectly synchronized, the signal $u[n]$ can be viewed as passing through an effective channel, whose impulse response is written as

$$
c[n]=\sum_{l=0}^{L_{f}-1} f[l] h[n-l], n=0,1, \cdots, L_{c}-1
$$

where $L_{c}=L_{f}+L_{h}$ is the length of the effective channel. Here, the CP length is assumed to be no smaller than the effective channel length, i.e., $L \geq L_{c}$, so that the ISI can be eliminated at the receiver [21].

\subsection{Receiver structure}

The modified zero-forcing receiver presented in [12] is adopted. Specifically, channel equalization in this paper is only performed on the subcarriers in the allocated subband, rather than on all the subcarriers, as presented in [12]. The block diagram of the receiver is depicted in Fig. 2. As suggested in [21], receiver filtering in the original filtering-based waveform is not considered here in order to scale down the complexity of the receiver.

The received signal after serial-to-parallel $(\mathrm{S} / \mathrm{P})$ and $\mathrm{CP}$ removal is multiplied by the DFT matrix $\mathbf{W}_{M}$, and then channel equalization for signals on the used subcarriers is performed by multiplying the inverse of the diagonal matrix $\boldsymbol{\Lambda}(\mathcal{K})$. After that, the equalized signal is multiplied by the de-windowing matrix $\mathbf{F}(\mathcal{K})$ in order to restore the orthogonality between subcarriers in the subband. In [12], it has been proven that the de-windowing matrix $\mathbf{F}(\mathcal{K})$ is independent of the effective channel if the window satisfies the cyclic-prefixed property: $w_{m}=w_{m+M}, m=-1,-2, \cdots,-L$. Based on this property, the windowed signal vector $\mathbf{u}[n]$ is mathematically equivalent to:

$$
\mathbf{u}[n]=\mathbf{R D} \mathbf{u}_{M}[n]
$$

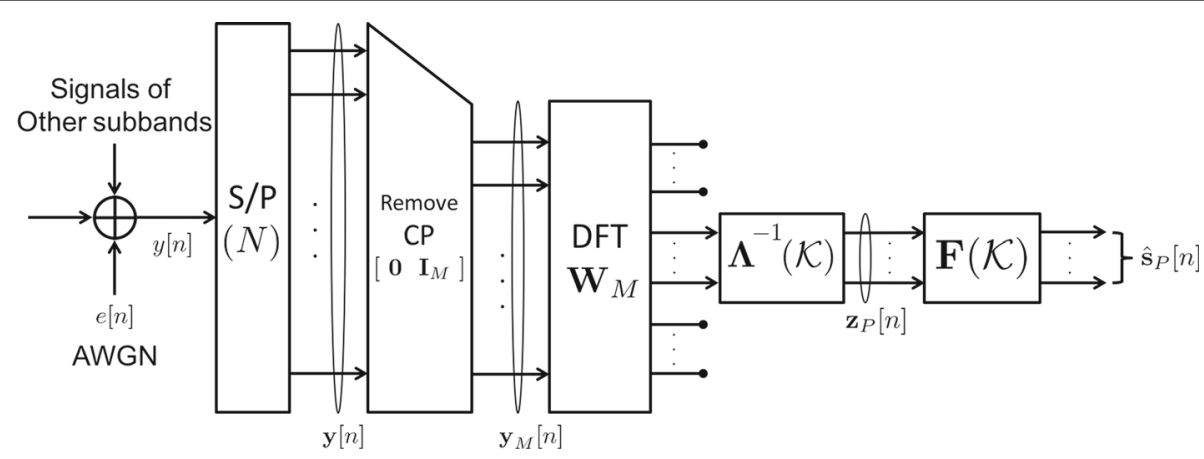

Fig. 2 Block diagram of the receiver 
where $\mathbf{D}=\operatorname{diag}(\mathbf{w})$ with $\mathbf{w} \triangleq\left[w_{0} w_{1} \cdots w_{M-1}\right]^{T}$. Without regarding the signals of users allocated to other subbands, it can be shown that the vector $\mathbf{y}_{M}[n]$, after serial-to-parallel conversion and CP removal, is

$$
\mathbf{y}_{M}[n]=\mathbf{C D} \mathbf{u}_{M}[n]+\mathbf{e}_{M}[n]
$$

where $\mathbf{C}$ is an $M \times M$ circulant matrix whose first column is $\left[c[0] c[1] \cdots c\left[L_{c}-1\right] \mathbf{0}_{\left(M-L_{c}-1\right)}\right]^{T}$ and $\mathbf{e}_{M}[n] \sim C N\left(\mathbf{0}_{M}, \sigma_{e}^{2} \mathbf{I}_{M}\right)$ is the additive white Gaussian noise vector [28]. The circulant matrix can be diagonalized by the DFT matrix:

$$
\mathbf{C}=\mathbf{W}_{M}^{H} \Lambda \mathbf{W}_{M}
$$

where $\Lambda=\operatorname{diag}\left(\left[C\left(e^{j 0}\right) C\left(e^{j \frac{2 \pi}{M}}\right) \cdots C\left(e^{j \frac{2 \pi}{M}(M-1)}\right)\right]\right)$ with $C\left(e^{j \omega}\right)$ denoting the effective channel frequency response. Since the user is allocated to the subband consisting of subcarriers in $\mathcal{K}$, equalization for the effective channel is only performed on the allocated subcarriers. Assuming that the receiver has perfect knowledge of effective channel responses of the allocated subcarriers, the equalized vector $\mathbf{z}_{P}[n]$ is expressed as

$$
\mathbf{z}_{P}[n]=\boldsymbol{\Lambda}^{-1}(\mathcal{K}) \mathbf{E}(\mathcal{K}) \mathbf{W}_{M} \mathbf{y}_{M}[n]
$$

where $\mathbf{E}(\mathcal{K})=\left[\begin{array}{lll}\mathbf{0}_{P \times(K-1)} & \mathbf{I}_{P} & \mathbf{0}_{P \times(M-P-K+1)}\end{array}\right]$, and $\boldsymbol{\Lambda}(\mathcal{K})$ is the $P \times P$ diagonal matrix whose diagonal elements are the effective channel frequency responses of subcarriers in $\mathcal{K}$. That is, the diagonal matrix is written as $\boldsymbol{\Lambda}(\mathcal{K})=$ $\operatorname{diag}\left(\left[C\left(e^{j \frac{2 \pi}{M} K}\right) C\left(e^{j \frac{2 \pi}{M}(K+1)}\right) \cdots C\left(e^{j \frac{2 \pi}{M}(K+P-1)}\right)\right]\right)$.

After equalization, to restore the orthogonality between subcarriers in the subband, de-windowing is performed so that the decoded symbol vector $\hat{\mathbf{s}}_{P}[n]$ is written as

$$
\hat{\mathbf{s}}_{P}[n]=\mathbf{F}(\mathcal{K}) \mathbf{z}_{P}[n] .
$$

where $\mathbf{F}(\mathcal{K}) \triangleq \mathbf{E}(\mathcal{K}) \mathbf{F E}^{T}(\mathcal{K})$. The matrix $\mathbf{F}$ is designed in a zero-forcing sense, which can be interpreted as transforming the equalized signal into a time-domain signal, by multiplying a diagonal matrix whose diagonal elements are the inverse of window coefficients, and then taking DFT on the product [12], i.e.,

$$
\mathbf{F}=\mathbf{W}_{M} \mathbf{D}^{-1} \mathbf{W}_{M}^{H} .
$$

Such de-windowing operation will cause SNR loss at the receiver with an arbitrary $\mathbf{w}$ other than a constantmodulus window, e.g., $\mathbf{w}=\mathbf{1}_{M}$.

A different receiver structure that adopts de-windowing before channel equalization was proposed in [13]. This structure is not considered here because the adopted one assures orthogonality between subcarriers while data reception is executed at the receiver, and ISI is eliminated given that $L \geq L_{c}$. Moreover, the adopted structure is more suitable for performing window optimization because SNR loss caused by noise enhancement can be quantified explicitly, which will be presented in Section 2.4.

\subsection{Noise analysis}

In this subsection, the SNR loss caused by de-windowing is derived, which plays an important role in window optimization. The derived SNR loss can be approximated as the same as that quantified in [12]. By substituting (8), (9), and $\mathbf{F}(\mathcal{K})$ in (11) into (10), the correlation matrix of vector $\mathbf{z}_{P}[n]$, after CP removal, DFT, and channel equalization, is written as

$$
\begin{aligned}
\mathbf{R}_{z} & =E\left\{\mathbf{z}_{P}[n] \mathbf{z}_{P}^{H}[n]\right\} \\
& =\mathbf{E}(\mathcal{K}) \mathbf{W}_{M} \mathbf{D} E\left\{\mathbf{u}_{M}[n] \mathbf{u}_{M}^{H}[n]\right\} \mathbf{D W}_{M}^{H} \mathbf{E}^{T}(\mathcal{K})+\Sigma_{z}
\end{aligned}
$$

where $\Sigma_{z}=\operatorname{diag}\left(\left[\sigma_{z_{1}}^{2} \sigma_{z_{2}}^{2} \cdots \sigma_{z_{P}}^{2}\right]\right)$ is the correlation matrix of noise after DFT and channel equalization. The $i$ th diagonal element of $\Sigma_{z}$ is written as

$$
\sigma_{z_{i}}^{2}=\frac{\sigma_{e}^{2}}{\left|C\left(e^{j \frac{2 \pi}{M}(i+K-1)}\right)\right|^{2}}=\frac{\sigma_{i}^{2}}{\left|F\left(e^{j \frac{2 \pi}{M}(i+K-1)}\right)\right|^{2}}
$$

where $\sigma_{i}^{2} \triangleq \sigma_{e}^{2} /\left|H\left(e^{j \frac{2 \pi}{M}(i+K-1)}\right)\right|^{2}$ is the resulting noise variance of the conventional OFDM system. After dewindowing is performed as in (11), the correlation matrix of $\hat{\mathbf{s}}_{P}[n]$ is written as $\mathbf{R}_{\hat{s}}=E_{s} \mathbf{I}_{P}+\mathbf{F}(\mathcal{K}) \Sigma_{z} \mathbf{F}^{H}(\mathcal{K})$. The output noise power of an entry in $\hat{s}_{P}[n]$ is the corresponding diagonal element of the latter term. After the de-windowing operation in (12), the total output noise power is written as

$$
\mathcal{N}_{\mathrm{WF}}=\sum_{i=1}^{P} \sigma_{z_{i}}^{2} \frac{1}{M} \sum_{m=0}^{M-1} \frac{1}{\left|w_{m}\right|^{2}} .
$$

As a special case of the proposed waveform, filteringbased waveforms apply rectangular windowing. The resulting total output noise power is $\mathcal{N}_{\mathrm{F}}=\sum_{i=1}^{P} \sigma_{z_{i}}^{2}$, which is obtained by substituting $w_{m}=1, \forall m \in$ $\{0, \cdots, M-1\}$ into (14). By letting $F\left(e^{j \frac{2 \pi}{M}(i+K-1)}\right)=$ $1, \forall i \in\{1, \cdots, P\}$ and adopting rectangular windowing, the total output noise of the conventional OFDM system is obtained as $\mathcal{N}_{\mathrm{C}}=\sum_{i=1}^{P} \sigma_{i}^{2}$.

To quantify the noise enhancement effect caused by dewindowing at the receiver compared to the conventional OFDM system, the SNR loss is defined as

$$
\sigma(\mathbf{w})=\frac{\mathcal{N}_{\mathrm{WF}}}{\mathcal{N}_{\mathrm{C}}}=\frac{\mathcal{N}_{\mathrm{WF}} / \mathcal{N}_{\mathrm{F}}}{\mathcal{N}_{\mathrm{C}} / \mathcal{N}_{\mathrm{F}}}=\frac{1}{M} \sum_{m=0}^{M-1} \frac{1}{\left|w_{m}\right|^{2}} \frac{\mathcal{N}_{\mathrm{F}}}{\mathcal{N}_{\mathrm{C}}} .
$$

As suggested in [20], a large variation of $\left|F\left(e^{j \Omega_{k}}\right)\right|^{2}, k \in$ $\mathcal{K}$ will degrade the bit error rate (BER) performance. Therefore, the passband ripple of transmit filtering is constrained tightly so that $\left|F\left(e^{j \Omega_{k}}\right)\right|^{2} \approx 1, \forall k \in \mathcal{K}$. Accordingly, the ratio of total noise power of filtering-based waveforms to the total noise power of the conventional OFDM is approximated as 


$$
\mathcal{R}=\frac{\mathcal{N}_{\mathrm{F}}}{\mathcal{N}_{\mathrm{C}}} \approx 1
$$

since $\sigma_{i}^{2} \approx \sigma_{z_{i}}^{2}, i=1, \cdots, P$. As a result, the SNR loss in (15) is approximated as

$$
\sigma(\mathbf{w}) \approx \frac{1}{M} \sum_{m=0}^{M-1} \frac{1}{\left|w_{m}\right|^{2}} .
$$

In the sequel, it is assumed that the approximation in (16) holds given a tight passband ripple constraint. The reasons are that (i) this approximation is accurate for most of the channel responses and (ii) the approximation error does not affect the BER performance of the proposed waveform, as will be shown later in Sections 5.3 and 5.7.

\section{Problem statement}

The goal of this paper is to jointly design the filter $\mathbf{f}$ and the window $\mathbf{w}$ so that the OOSBE can be suppressed with a controllable SNR loss, and the passband ripple caused by filtering is restrained. The design is based on a minimax criterion. In [29], it has been pointed out that a wide transition bandwidth (TBW) is beneficial to suppressing stopband magnitude for minimax-based filter design with specified filter length and passband ripple. This phenomenon suggests that the OOSBE suppression performance of a filtering-based waveform is limited with a fixed number of VCs, filter length, and passband ripple. Here, windowing is introduced to further improve the OOSBE suppression performance over the filtering-based waveforms.

Suppose a user is allocated with a subband $\left(\omega_{\mathrm{p}}^{(l)}, \omega_{\mathrm{p}}^{(r)}\right)$. Then, the specification of the transmit PSD is illustrated in Fig. 3. Let the stopband and the passband of the filter and the window be defined as $B_{\mathrm{s}} \triangleq\left[-\pi, \omega_{\mathrm{s}}^{(l)}\right] \cup\left[\omega_{\mathrm{s}}^{(r)}, \pi\right)$ and $B_{\mathrm{p}} \triangleq\left(\omega_{\mathrm{p}}^{(l)}, \omega_{\mathrm{p}}^{(r)}\right)$, respectively. Neighboring subbands allocated to other users lie in the stopband such that the ICI between users can be mitigated. Define $B_{\mathrm{t}} \triangleq$ $\left(\omega_{\mathrm{s}}^{(l)}, \omega_{\mathrm{p}}^{(l)}\right] \cup\left[\omega_{\mathrm{p}}^{(r)}, \omega_{\mathrm{s}}^{(r)}\right)$ as the transition band. The subcarriers in the transition band are treated as VCs. Let $\eta$ denote the one-side normalized TBW, which is expressed as half the TBW over the subcarrier spacing (i.e., $2 \pi / M$ ), then the set of left and the right edges of the stopband are

$$
\left(\omega_{\mathrm{s}}^{(l)}, \omega_{\mathrm{s}}^{(r)}\right)=\left(\omega_{\mathrm{p}}^{(l)}-\frac{(2 \eta+1) \pi}{M}, \omega_{\mathrm{p}}^{(r)}+\frac{(2 \eta+1) \pi}{M}\right),
$$

where $\left(\omega_{\mathrm{p}}^{(l)}, \omega_{\mathrm{p}}^{(r)}\right)=\left(\omega_{\mathrm{c}}-P \pi / M, \omega_{\mathrm{c}}+P \pi / M\right)$. For convenience, $\eta$ can be thought of as the number of VCs on one side of the transition band.

Our design objective is to minimize the maximum (weighted) PSD in the stopband. The minimization is subject to constraints of SNR loss and passband ripple. In addition, for fair comparison, the signals after windowing and filtering should not have any power amplification effect. Therefore, the constraints (3) and (5) should be satisfied. Based on the above requirements, the optimization problem is expressed as

$$
\min _{\mathbf{w}, \mathbf{f}} \max _{\omega \in B_{\mathrm{s}}} G\left(e^{j \omega}\right) \mathcal{S}_{x}\left(e^{j \omega}\right)
$$

subject to $1 / \alpha \leq\left|F\left(e^{j \Omega_{k}}\right)\right|^{2} \leq \alpha, \forall k \in \mathcal{K}$

$$
\begin{aligned}
& \int_{-\pi}^{\pi} \mathcal{S}_{x}\left(e^{j \omega}\right) \frac{\mathrm{d} \omega}{2 \pi}=\int_{-\pi}^{\pi} \mathcal{S}_{u}\left(e^{j \omega}\right) \frac{\mathrm{d} \omega}{2 \pi} \\
& \sigma(\mathbf{w}) \leq \gamma \\
& \sum_{m=-L}^{M-1}\left|w_{m}\right|^{2}=N
\end{aligned}
$$

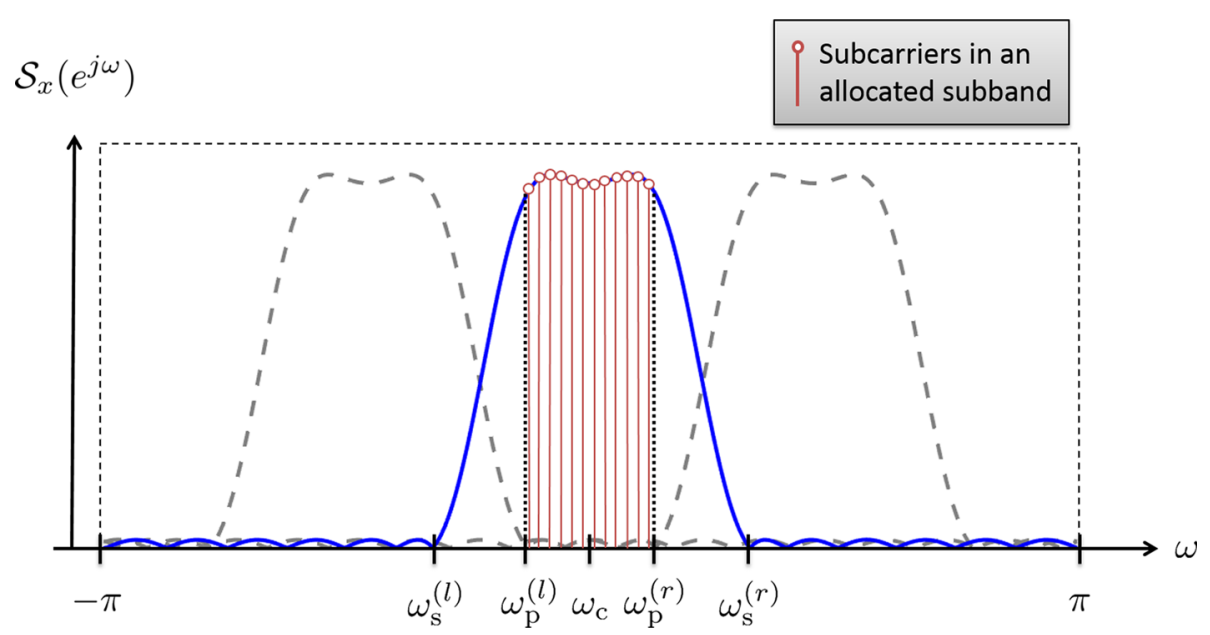

Fig. 3 An illustration of problem statement 
where $\alpha$ and $\gamma$ denote controllable parameters of passband ripple and SNR loss, respectively, and $G\left(e^{j \omega}\right)$ is the weighting function of the stopband in order to meet a specified spectrum mask restriction. For simplicity, it is assumed that $G\left(e^{j \omega}\right)=1, \forall \omega \in B_{\mathrm{s}}$ in the following derivations. When a more general $G\left(e^{j \omega}\right)$ is considered, however, it may not impose too much difficulty in generalizing the derivations.

Here, problem (19) is formulated as a more compact form. Based on the cyclic-prefix window property, the Fourier transform of window coefficients in (1) can be written as $W\left(e^{j \omega}\right)=\mathbf{v}_{N}^{H}\left(e^{j \omega}\right) \mathbf{R w}$. Consequently, the PSD of signal $u[n]$ is expressed as a quadratic form:

$$
\mathcal{S}_{u}\left(e^{j \omega}\right)=\mathbf{w}^{H} \mathbf{B}\left(e^{j \omega}\right) \mathbf{w}
$$

where the positive semidefinite matrix $\mathbf{B}\left(e^{j \omega}\right)$ is

$$
\mathbf{B}\left(e^{j \omega}\right)=\frac{E_{s}}{N} \mathbf{R}^{T} \sum_{k \in \mathcal{K}} \mathbf{v}_{N}\left(e^{j\left(\omega-\Omega_{k}\right)}\right) \mathbf{v}_{N}^{H}\left(e^{j\left(\omega-\Omega_{k}\right)}\right) \mathbf{R}
$$

With this formulation, the original problem (19) is written as

$$
\begin{gathered}
\min _{\mathbf{w}, \mathbf{f}} \max _{\omega \in B_{\mathrm{s}}} \mathbf{w}^{H} \mathbf{B}\left(e^{j \omega}\right) \mathbf{w}\left|\mathbf{v}_{L_{f}}^{H}\left(e^{j \omega}\right) \mathbf{f}\right|^{2} \\
\text { subject to } 1 / \alpha \leq\left|\mathbf{v}_{L_{f}}^{H}\left(e^{j \Omega_{k}}\right) \mathbf{f}\right|^{2} \leq \alpha, \forall k \in \mathcal{K} \\
\int_{-\pi}^{\pi} \mathbf{w}^{H} \mathbf{B}\left(e^{j \omega}\right) \mathbf{w}\left|\mathbf{v}_{L_{f}}^{H}\left(e^{j \omega}\right) \mathbf{f}\right|^{2} \frac{\mathrm{d} \omega}{2 \pi}=P E_{s} \\
\sigma(\mathbf{w}) \leq \gamma \\
\|\mathbf{R} \mathbf{w}\|^{2}=N .
\end{gathered}
$$

This problem is nonconvex due to, e.g., constraints (22b), (22c), or (22e) [30]. Therefore, the well-developed convex optimization techniques [30] can not be applied directly to solve this problem. Instead of optimizing $\mathbf{w}$ and f simultaneously, a method is proposed to optimize $\mathbf{w}$ and f iteratively.

\section{Proposed method}

In this section, an alternative optimization method to solve problem (19) is proposed. First, the problem (22) is divided into two subproblems, which are (i) optimization of filter: optimize the filter function $\mathbf{f}$, subject to filter constraints with a given window, and (ii) optimization of window: optimize the cyclic-prefixed window coefficients $w_{m}, m=-L, \cdots, M-1$, subject to window constraints with a given filter. Then, an iterative algorithm is proposed to solve the original optimization problem. The convergence and computational complexity of the proposed method, and the complexity of the transceiver are analyzed.

\subsection{Optimization of filter function}

The filter design method presented in [20] is used by relaxing the nonconvex problem into a convex one using spectral factorization [31]. The relaxed problem can be solved by using an interior-point method (IPM) in [30]. Given any $\mathbf{w}$ that satisfies (22d) and (22e), problem (22) becomes a filter optimization problem written as

$$
\begin{gathered}
\min _{\mathbf{f}} \max _{\omega \in B_{\mathrm{s}}} \mathbf{w}^{H} \mathbf{B}\left(e^{j \omega}\right) \mathbf{w}\left|\mathbf{v}_{L_{f}}^{H}\left(e^{j \omega}\right) \mathbf{f}\right|^{2} \\
\text { subject to } 1 / \alpha \leq\left|\mathbf{v}_{L_{f}}^{H}\left(e^{j \Omega_{k}}\right) \mathbf{f}\right|^{2} \leq \alpha, k \in \mathcal{K} \\
\int_{-\pi}^{\pi} \mathbf{w}^{H} \mathbf{B}\left(e^{j \omega}\right) \mathbf{w}\left|\mathbf{v}_{L_{f}}^{H}\left(e^{j \omega}\right) \mathbf{f}\right|^{2} \frac{\mathrm{d} \omega}{2 \pi}=P E_{s} .
\end{gathered}
$$

This problem is nonconvex due to the lower-bounded constraint (23b) and the equality constraint (23c). This issue is overcome by using a technique presented in [32]. Let the auto-correlation function of $f[n]$ be defined as

$$
r_{f}(m)=\sum_{n=0}^{L_{f}-1} f[n] f^{*}[n+m] .
$$

Then, the squared magnitude of $F\left(e^{j \omega}\right)$ can be written as

$$
\left|F\left(e^{j \omega}\right)\right|^{2}=\mathbf{a}^{T}\left(e^{j \omega}\right) \mathbf{r}_{f} \geq 0
$$

where $\mathbf{r}_{f}=\left[r_{f}\left(-L_{f}+1\right) \cdots r_{f}(0) \cdots r_{f}\left(L_{f}-1\right)\right]^{T}$ and

$$
\mathbf{a}\left(e^{j \omega}\right)=\left[\begin{array}{lllll}
e^{j\left(L_{f}-1\right) \omega} & \cdots & 1 & \cdots & e^{-j\left(L_{f}-1\right) \omega}
\end{array}\right]^{T} .
$$

Note that $r_{f}(m)$ is conjugate-symmetric, i.e., $r_{f}(m)=$ $r_{f}^{*}(-m)$. With (24), the PSD in (4) can be rewritten as

$$
\mathcal{S}_{x}\left(e^{j \omega}\right)=\mathbf{w}^{H} \mathbf{B}\left(e^{j \omega}\right) \mathbf{w a}^{T}\left(e^{j \omega}\right) \mathbf{r}_{f} .
$$

With this equation and some manipulations, the problem (23) is modified as

$$
\begin{gathered}
\min _{\mathbf{r}_{f}} \max _{\omega \in B_{\mathrm{s}}} \mathbf{b}^{T}\left(e^{j \omega}\right) \mathbf{r}_{f} \\
\text { subject to } 1 / \alpha \leq \mathbf{a}^{T}\left(e^{j \Omega_{k}}\right) \mathbf{r}_{f} \leq \alpha, k \in \mathcal{K} \\
\mathbf{q}^{T} \mathbf{r}_{f}=P N \\
\mathbf{a}^{T}\left(e^{j \omega}\right) \mathbf{r}_{f} \geq 0, \forall \omega \in[-\pi, \pi) \\
r_{f}(m)=r_{f}^{*}(-m), m=0,1, \cdots, L_{f}-1
\end{gathered}
$$

where $\mathbf{b}\left(e^{j \omega}\right)=\mathbf{w}^{H} \mathbf{B}\left(e^{j \omega}\right) \mathbf{w} \mathbf{a}^{T}\left(e^{j \omega}\right)$ and the vector $\mathbf{q}$ is given by $[\mathbf{q}]_{i}=\mathbf{w}^{H} \mathbf{R}^{T} \mathbf{Q}_{i} \mathbf{R} \mathbf{w}$ with

$$
\left[\mathbf{Q}_{i}\right]_{m, n}= \begin{cases}\sum_{k \in \mathcal{K}} e^{j(n-m) \Omega_{k}}, & m-n+L_{f}-i=0 \\ 0, & \text { else. }\end{cases}
$$

Details of the derivation of constraint (27c) from (23c) are described in Appendix 1. The problem (27) is semiinfinite, which means that there are infinite number of 
constraints. A straightforward way of relaxing this problem is to discretize $\omega$ by sampling a finite set of frequencies in $[-\pi, \pi)$ such that

$$
-\pi \leq \check{\omega}_{0}<\cdots<\check{\omega}_{J-1}<\pi
$$

where $J$ denotes the number of samples. This paper adopts uniformly spaced sampling, i.e., $\check{\omega}_{i}=-\pi+2 \pi i / J, i=$ $0,1, \cdots, J-1$. A previous work [33] recommended that $J \approx 30 L_{f}$ is sufficiently large to approximate the problem (27). With this discretization, the filter optimization problem (27) is modified as

$$
\min _{\mathbf{r}_{f}} \max _{\check{\omega}_{i} \in \check{B}_{s}} \mathbf{b}^{T}\left(e^{j \check{\omega}_{i}}\right) \mathbf{r}_{f}
$$

subject to $1 / \alpha \leq \mathbf{a}^{T}\left(e^{j \Omega_{k}}\right) \mathbf{r}_{f} \leq \alpha, k \in \mathcal{K}$

$$
\begin{aligned}
& \mathbf{q}^{T} \mathbf{r}_{f}=P N \\
& \mathbf{a}^{T}\left(e^{j \check{\omega}_{i}}\right) \mathbf{r}_{f} \geq 0, \forall \check{\omega}_{i} \in \check{B}_{\mathrm{a}} \\
& r_{f}(m)=r_{f}^{*}(-m), m=0,1, \cdots, L_{f}-1
\end{aligned}
$$

where $\check{B}_{\mathrm{S}}=\left\{\check{\omega}_{i} \mid \check{\omega}_{i}=-\pi+2 \pi i / J, i=0,1, \cdots, J-1\right.$, and $\left.\check{\omega}_{i} \in B_{\mathrm{s}}\right\}$ and $\check{B}_{\mathrm{a}}=\left\{\check{\omega}_{i} \mid \check{\omega}_{i}=-\pi+2 \pi i / J, i=0\right.$, $1, \cdots, J-1\}$. This problem can be solved efficiently by existing toolboxes (e.g., CVx [34]). To obtain $\mathbf{f}$ from the optimal $\mathbf{r}_{f}$, spectral factorization in [31] can be applied using an efficient implementation based on inverse fast Fourier transform (IFFT) and fast Fourier transform (FFT).

\subsection{Optimization of window coefficients}

A per-subband optimization method is proposed that minimizes the maximum stopband PSD subject to window constraints in (22), whereas per-subcarrier optimization was proposed in [12]. Specifically, the window coefficients are restricted as real, and then the window optimization problem is relaxed as a convex one. By adopting the technique presented in Section 4.1, problem (22) is reformulated as

$$
\begin{gathered}
\min _{\mathbf{w}, \mathbf{r}_{f}} \max _{\omega \in B_{\mathrm{s}}} \mathbf{w}^{H} \mathbf{B}\left(e^{j \omega}\right) \mathbf{w} \mathbf{a}^{T}\left(e^{j \omega}\right) \mathbf{r}_{f} \\
\text { subject to } 1 / \alpha \leq \mathbf{a}^{T}\left(e^{j \Omega_{k}}\right) \mathbf{r}_{f} \leq \alpha, \forall k \in \mathcal{K} \\
\int_{-\pi}^{\pi} \mathbf{w}^{H} \mathbf{B}\left(e^{j \omega}\right) \mathbf{w} \mathbf{a}^{T}\left(e^{j \omega}\right) \mathbf{r}_{f} \frac{\mathrm{d} \omega}{2 \pi}=P E_{s} \\
\mathbf{a}^{T}\left(e^{j \omega}\right) \mathbf{r}_{f} \geq 0, \forall \omega \in[-\pi, \pi) \\
r_{f}(m)=r_{f}^{*}(-m), m=0,1, \cdots, L_{f}-1 \\
\\
\sigma(\mathbf{w}) \leq \gamma \\
\|\mathbf{R} \mathbf{w}\|^{2}=N .
\end{gathered}
$$

Given any fixed $\mathbf{r}_{f}$ that satisfies (31b), (31d), and (31e). The constraint (31c) is removed and the problem (22) is rewritten as

$$
\begin{gathered}
\min _{\mathbf{w}} \max _{\omega \in B_{\mathbf{s}}} \mathbf{w}^{H} \mathbf{U}\left(e^{j \omega}\right) \mathbf{w} \\
\text { subject to } \sigma(\mathbf{w}) \leq \gamma \\
\|\mathbf{R} \mathbf{w}\|^{2}=N .
\end{gathered}
$$

where $\mathbf{U}\left(e^{j \omega}\right)=\mathbf{B}\left(e^{j \omega}\right) \mathbf{a}^{T}\left(e^{j \omega}\right) \mathbf{r}_{f}$. The reasons that the constraint (31c) is removed are that (31c) is nonconvex in $\mathbf{w}$ in general, and the relaxation does not prevent us from finding the optimal $\mathbf{w}$ and $\mathbf{f}$ for problem (22), as will be explained in Section 4.3.

In the literature, a common way of dealing with a complex value optimization problem is to reformulate it as a real-valued one via separating the real part and imaginary part of the parameters (e.g., [35, 36]). Define $\hat{\mathbf{w}}=\left[\operatorname{Re}\left\{\mathbf{w}^{T}\right\} \operatorname{Im}\left\{\mathbf{w}^{T}\right\}\right]^{T}$, then the SNR loss in (32b) is expressed as a function of $\hat{\mathbf{w}}$ :

$$
\hat{\sigma}(\hat{\mathbf{w}})=\frac{1}{M} \sum_{m=1}^{M} \frac{1}{\hat{w}_{m}^{2}+\hat{w}_{m+M}^{2}}
$$

where $\hat{w}_{m} \triangleq[\hat{\mathbf{w}}]_{m}$. Subsequently, problem (32) is reformulated via separating the real and imaginary parts of $\mathbf{w}$, and relaxing (32c) as an inequality constraint so that this problem becomes a convex one. Then,

$$
\begin{gathered}
\min _{\hat{\mathbf{w}}} \max _{\omega \in B_{\mathrm{s}}} \hat{\mathbf{w}}^{T} \hat{\mathbf{U}}\left(e^{j \omega}\right) \hat{\mathbf{w}} \\
\text { subject to } \hat{\sigma}(\hat{\mathbf{w}}) \leq \gamma \\
\|\hat{\mathbf{R}} \hat{\mathbf{w}}\|^{2} \leq N
\end{gathered}
$$

where $\hat{\mathbf{R}} \triangleq \mathbf{1}_{2} \otimes \mathbf{R}$ and

$$
\hat{\mathbf{U}}\left(e^{j \omega}\right)=\left[\begin{array}{lr}
\operatorname{Re}\left\{\mathbf{U}\left(e^{j \omega}\right)\right\} & -\operatorname{Im}\left\{\mathbf{U}\left(e^{j \omega}\right)\right\} \\
\operatorname{Im}\left\{\mathbf{U}\left(e^{j \omega}\right)\right\} & \operatorname{Re}\left\{\mathbf{U}\left(e^{j \omega}\right)\right\}
\end{array}\right] .
$$

The convexity of this problem depends on constraint (34b), since the objective function and constraint (34b) are convex. The convexity of $\hat{\sigma}(\hat{\mathbf{w}}), \operatorname{dom} \hat{\sigma} \subseteq \mathrm{R}_{+}^{2 M}$ is shown by the following theorem.

\section{Theorem 1 Convexity of $\hat{\sigma}(\hat{\boldsymbol{w}})$ :}

(a) The function $\hat{\sigma}(\hat{\boldsymbol{w}})$ in (33) is convex if $\operatorname{dom} \hat{\sigma}=\mathrm{D}(\boldsymbol{\theta}) \triangleq\left\{\left(\mathbf{1}_{2} \otimes \boldsymbol{\tau}\right) \circ \boldsymbol{d}(\boldsymbol{\theta}) \mid \boldsymbol{\tau} \in \mathrm{R}_{++}^{M}\right\}$ for any $\boldsymbol{\theta}=\left[\theta_{1} \cdots \theta_{M}\right]^{T}$ that satisfies $\theta_{1}, \cdots, \theta_{M} \in[-\pi, \pi)$.

(b) The function $\hat{\sigma}$ is nonconvex if $\operatorname{dom} \hat{\sigma}=\mathrm{R}_{++}^{2 M}$.

Proof See Appendix 2.

Theorem 1 suggests that problem (34) is convex if $\operatorname{dom} \hat{\sigma}=\mathrm{D}(\boldsymbol{\theta})$ for any $\theta_{1}, \cdots, \theta_{M} \in[-\pi, \pi)$. For simplicity, restricting $\boldsymbol{\theta}=\mathbf{0}_{M}$ is adopted, in which case the window coefficients are positive-valued. This restriction 
implies that the original SNR loss function $\sigma(\mathbf{w}), \operatorname{dom} \sigma=$ $\mathrm{R}_{++}^{M}$, is convex, which is suggested by the proof of Theorem 1(a). By applying frequency discretization as in (29) and (30), problem (34) is approximated as

$$
\min _{\mathbf{w}} \max _{\check{\omega}_{i} \in \check{B}_{\mathrm{s}}} \mathbf{w}^{T} \mathbf{U}\left(e^{j \check{\omega}_{i}}\right) \mathbf{w}
$$

subject to $\sigma(\mathbf{w}) \leq \gamma$

$$
\mathbf{w}^{T} \mathbf{R}^{T} \mathbf{R} \mathbf{w} \leq N
$$

where $\mathbf{w} \in \mathrm{R}_{++}^{M}$. This problem is a convex optimization problem with finite constraints, which can be solved by using $\mathrm{Cvx}$.

\subsection{Proposed iterative method for joint optimization}

An iterative method that optimizes window and filter is proposed. In an iteration of the proposed method, the window optimization and filter optimization presented in the previous subsections are performed based on given filter function and given window coefficients, respectively. Specifically, window optimization is performed based on the optimal filter's auto-correlation function obtained in the previous iteration, and then filter optimization is performed based on the optimal window of the current iteration.

A detailed description of the proposed method is shown in Algorithm 1. In each iteration, the window optimization in (35) and the filter optimization (30) are performed sequentially. Let $\mathbf{w}(t)$ and $\mathbf{r}_{f}(t)$ denote the optimal solutions of (35) and (30) in iteration $t$, respectively. The squared differences of the optimal filter's correlation function vector and optimal window between iterations $t$ and $t-1$ are, respectively, written as $\xi_{w}(t)=\|\mathbf{w}(t)-\mathbf{w}(t-1)\|^{2}$ and $\xi_{r}(t)=\left\|\mathbf{r}_{f}(t)-\mathbf{r}_{f}(t-1)\right\|^{2}$. Since the proposed method is convergent (the proof will be given in Section 4.4), the squared differences $\xi_{w}(t)$ and $\xi_{r}(t)$ will decrease as the number of iterations increases. Therefore, the stopping criteria of the proposed iterative algorithm is that both $\xi_{w}(t)$ and $\xi_{r}(t)$ are smaller than pre-determined tolerances $\epsilon_{w}$ and $\epsilon_{r}$, respectively. Let $\hat{t}$ denote the termination iteration index. Then, the optimal window is $\mathbf{w}(\hat{t})$, and the optimal filter is obtained by performing spectral factorization [31] on $\mathbf{r}_{f}(\hat{t})$. For convenience, the starting point is given as $\mathbf{r}_{f}(0)=\left[\begin{array}{lll}\mathbf{0}_{L_{f}-1}^{T} & 1 & \mathbf{0}_{L_{f}-1}^{T}\end{array}\right]^{T}$, which means that no transmit filtering is deployed. Other starting points have been tried, and the resulting performances in OOSBE suppression are close to or worse than that of the current one. Finding the optimal starting point is an open problem for alternative optimization algorithms $[37,38]$, which is left for our future work.

In any iteration of the algorithm, the pair $\left(\mathbf{w}(t), \mathbf{r}_{f}(t)\right)$ must satisfy the constraint (27c) after filter optimization.
Accordingly, the constraint (31c) is not necessary to be met by the window $\mathbf{w}(t)$ based on $\mathbf{r}_{f}(t-1)$. Therefore, it is removed from (31) in window optimization (32).

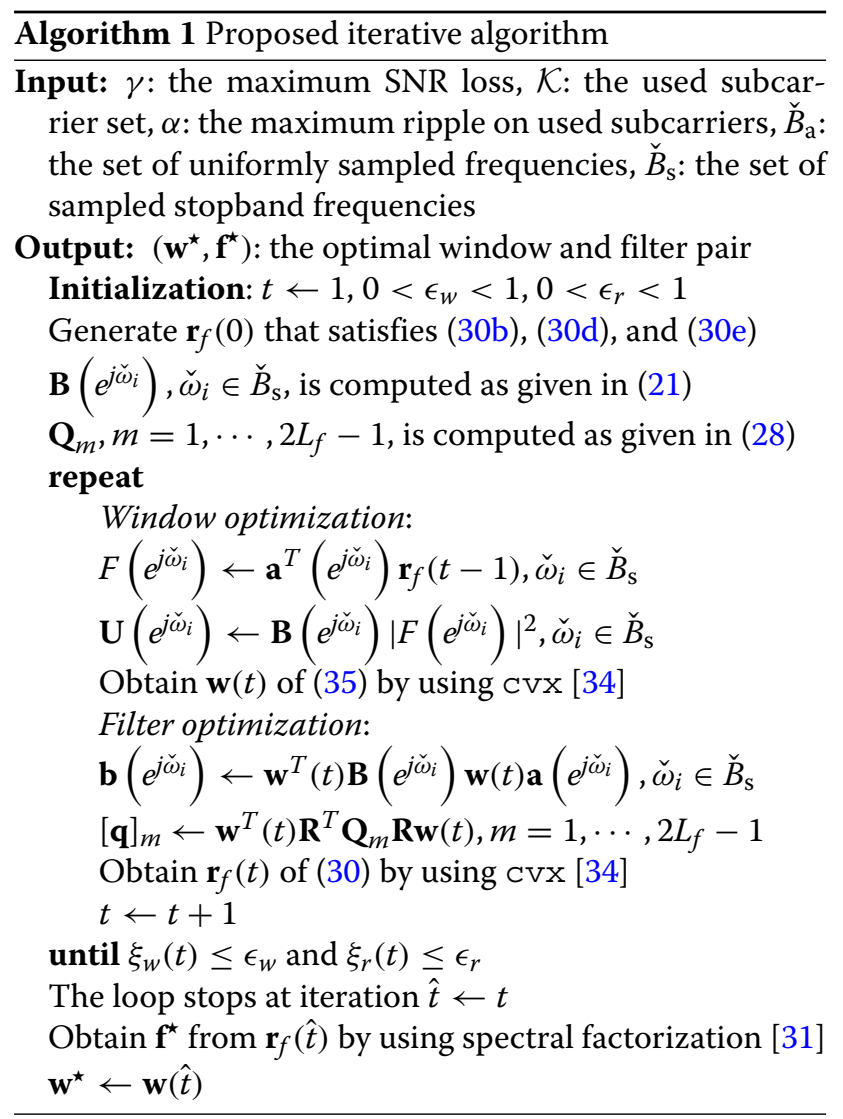

\subsection{Convergence analysis of the proposed algorithm}

It is necessary to characterize whether the proposed algorithm is convergent, since it is operated iteratively. To justify that the convergence of the proposed algorithm is guaranteed, it is sufficient to show that the iteration of updating $\mathbf{w}$ and $\mathbf{r}_{f}$ converges and the objective is lowerbounded. A similar argument can be found in [37, 38]. In each iteration, the optimal $\mathbf{w}$ is first obtained by solving (35). The optimal $\mathbf{r}_{f}$ is then computed by solving (30) based on the determined $\mathbf{w}$. Consequently, the objective function $\max _{\check{\omega}_{i} \in \check{B}_{\mathrm{s}}} \mathbf{w}^{H} \mathbf{B}\left(e^{j \check{\omega}_{i}}\right) \mathbf{w} \mathbf{a}^{T}\left(e^{j \check{\omega}_{i}}\right) \mathbf{r}_{f}$ decreases in each iteration. Clearly, the objective function is lowerbounded because the stopband PSD is non-negative. Therefore, the proposed algorithm is convergent.

\subsection{Complexity analysis of the proposed algorithm}

The computational complexity of the IPM [30] for problem (30) is expressed as $O\left(L_{f}^{3.5}\right)$ [36]. The complexity order of spectral factorization for obtaining the 
optimal filter from its auto-correlation function using an FFT-based implementation is $O\left(L_{f} \log _{2} L_{f}\right)$ [31]. Similar to filter optimization, the computational complexity of the IPM for window optimization is expressed as $O\left(M^{3.5}\right)$ [39]. As a result, the computational complexity in each iteration of the proposed iterative algorithm is $O\left(M^{3.5}+L_{f}^{3.5}+L_{f} \log _{2} L_{f}\right)$, which is equivalent to $O\left(M^{3.5}\right)$ since $L_{f} \ll M$. Observe that the number of iterations for satisfying $\xi_{w}(t) \leq \epsilon_{w}$ and $\xi_{f}(t) \leq$ $\epsilon_{r}$ is independent from $M$. Therefore, the complexity order of the proposed method can also be expressed as $O\left(M^{3.5}\right)$.

\subsection{Complexity analysis of the transceiver}

At the transmitter, the number of arithmetic operators of cyclic-prefixed windowing is expressed as $O(M)$ because the windowed signal can be represented by (7); the number of arithmetic operators of filtering is expressed as $O\left(N L_{f}\right)=O\left(N^{2}\right)$ since $L_{f}$ is usually an integer equal to $N$ divided by a power of two and can possibly be reduced to $O\left(N \log _{2} N\right)$ [40]. De-windowing at the receiver in (11) can be implemented by using FFT, IFFT, and $M$ additional multiplications. Therefore, the computational complexity of receiver with de-windowing is $O\left(3 M \log _{2} M+M\right)=$ $O\left(M \log _{2} M\right)$, which is of the same order as the receiver of conventional OFDM.

\section{Results and discussion}

In this section, some numerical results are provided for demonstrating the advantages of the proposed waveform.

\subsection{Parameter settings}

The parameters used in our simulations are listed below:

- Total number of subcarriers: $M=128$

- Number of subcarriers in a subband: $P=12$

- Maximum channel order: $L_{h}=M / 8=16$

- Filter length: $L_{f}=17$

- CP length: $L=M / 4=32$

The values of the channel impulse response in (6) are assumed to be i.i.d. complex Gaussian random variables:

$$
h[n] \sim C N(0,1), n=0,1, \cdots, L_{h} .
$$

There are $10^{7}$ channel realizations used in our simulations. It is assumed that all subcarriers in the subband are used, i.e., $\mathcal{K}=\{0,1, \cdots, P-1\}$. The center frequency of the allocated subband is $\omega_{\mathrm{c}}=(P-1) \pi / M$. The passband set $\left(\omega_{\mathrm{p}}^{(l)}, \omega_{\mathrm{p}}^{(r)}\right)$, and the pair stopband edges, $\omega_{\mathrm{s}}^{(l)}$ and $\omega_{\mathrm{s}}^{(r)}$, are computed as Eq. (18) with arbitrary normalized TBW $\eta$.
In the following simulations, the proposed waveform is referred to as "joint windowing and filtering" (joint W-F).

\subsection{Convergence of the proposed algorithm}

Figure 4 verifies the convergence of the proposed optimization method under different configurations. The normalized TBW used in this figure is $\eta=3.5$. The left and right plots demonstrate that the objective function in (22) decreases and converges rapidly in each iteration after window optimization and filter optimization, respectively.

\subsection{Approximation of SNR loss}

To examine the accuracy of the approximation in (17) with respect to various passband ripple constraints, the histogram of the ratio $\mathcal{R}$ in (16), based on $10^{7}$ arbitrary channel realizations, is depicted in Fig. 5, and the probability of the ratio falling in a range is shown in Table 1. The normalized TBW used here is also $\eta=$ 3.5. One can observe that the ratios with $\alpha=0.3 \mathrm{~dB}$ and $\alpha=0.5 \mathrm{~dB}$ both have peak probabilities very close to $\mathcal{R}=1$. In addition, over $96 \%$ of the ratios with $\alpha=0.3 \mathrm{~dB}$ and $\alpha=0.5 \mathrm{~dB}$ distribute in the range of $(-0.93,1.07)$. These observations suggest that the approximation is tight for most of the channel realizations, and the approximation error imposes a slight affect on the BER performance, as will be shown in Section 5.7. On the contrary, the ratio with $\alpha=1 \mathrm{~dB}$ leads to inaccurate approximation since its distribution is scattered in a much wider range and does not have a peak probability close to $\mathcal{R}=1$. In summary, $\alpha=0.5 \mathrm{~dB}$ is chosen for the following simulations because it results in an accurate SNR loss approximation and has a better OOSBE suppression performance than the case with $\alpha=0.3 \mathrm{~dB}$.

\subsection{Related waveforms for comparison}

To highlight the advantages of the proposed joint W-F waveform, the following related waveforms that use either windowing or filtering are introduced for comparison in our simulations.

(a) Pure filtering: The waveform deploys optimum filtering in [20] at the transmitter with rectangular windowing.

(b) Pure windowing: The waveform deploys optimum windowing without filtering at the transmitter. The optimal window is obtained through solving (35).

(c) WOLA at the transmitter: Transmitter windowing of WOLA is performed on some of the CP symbol and an additional cyclic suffix (CS), and then the weighted CS is overlapped with the weighted CP of the next symbol. The transceiver structure in ([26], 

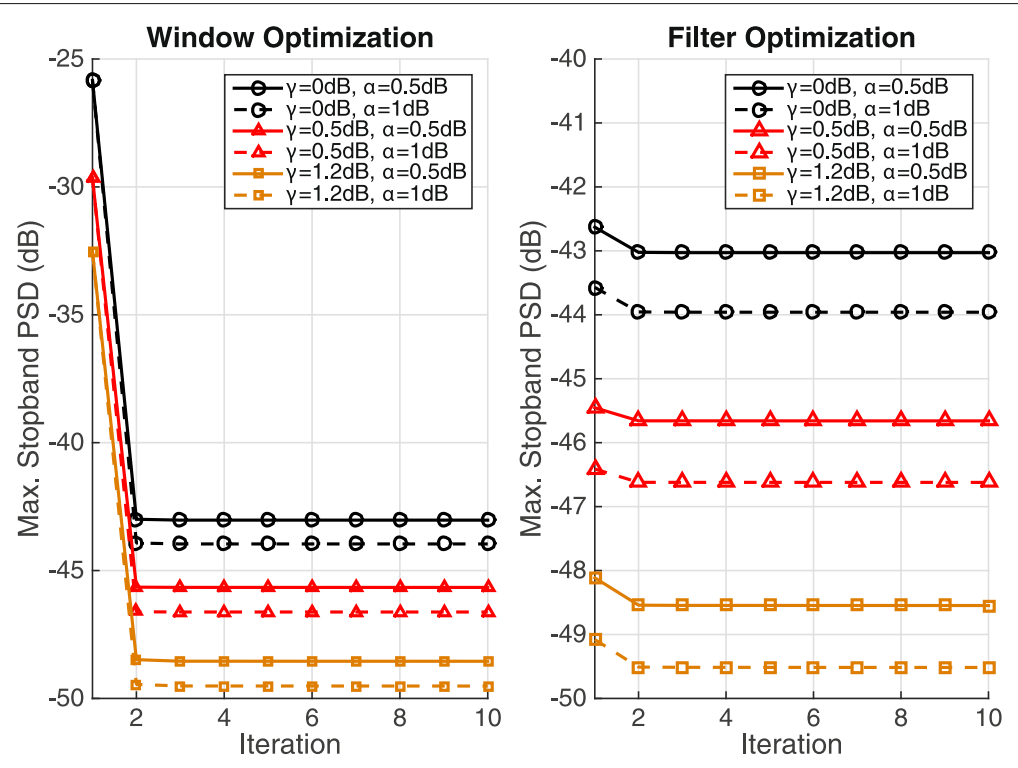

Fig. 4 Convergence of the proposed method

[Ch. 9]) is adopted for simulations. The raised-cosine (R-C) window is usually used by current WOLA systems, which is expressed as

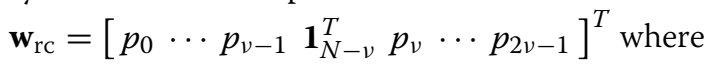

$$
p_{n}=0.5\left(1-\cos \left(\frac{n+1}{v} \pi\right)\right), n=0, \cdots, v-1
$$

and $p_{n}=1-p_{n-v}, n=v, \cdots, 2 v-1$ [41] with $v$ denoting the CS length. The receiver of WOLA is the same as that of the conventional OFDM system. To eliminate ISI introduced at the receiver, the CP length of WOLA should satisfy $L \leq L_{h}+v$. This paper assumes that $v=L_{f}$ for fair comparison.

\subsection{Normalized PSD}

Here, the PSDs of the transmitted signals modulated by the aforementioned waveforms are demonstrated. The PSD is normalized so that its value on an allocated subband approaches $0 \mathrm{~dB}$. The normalized PSD is written as

$$
\overline{\mathcal{S}}_{x}\left(e^{j \omega}\right)=\frac{1}{N E_{s}} \mathcal{S}_{x}\left(e^{j \omega}\right) \text {. }
$$

Figures 6 and 7 depict the normalized PSDs of the proposed and the other waveforms. In Fig. 6, the normalized TBWs of the pure filtering, pure windowing, and the proposed waveforms are $\eta=8.5$, which is as wide as that of WOLA. The resulting maximum stopband PSD of the proposed waveform with SNR loss $\gamma=1.2 \mathrm{~dB}$ is about $-67 \mathrm{~dB}$, which is the best performance compared to the others. With a little bit less SNR loss, the proposed waveform with $\gamma=0.5$

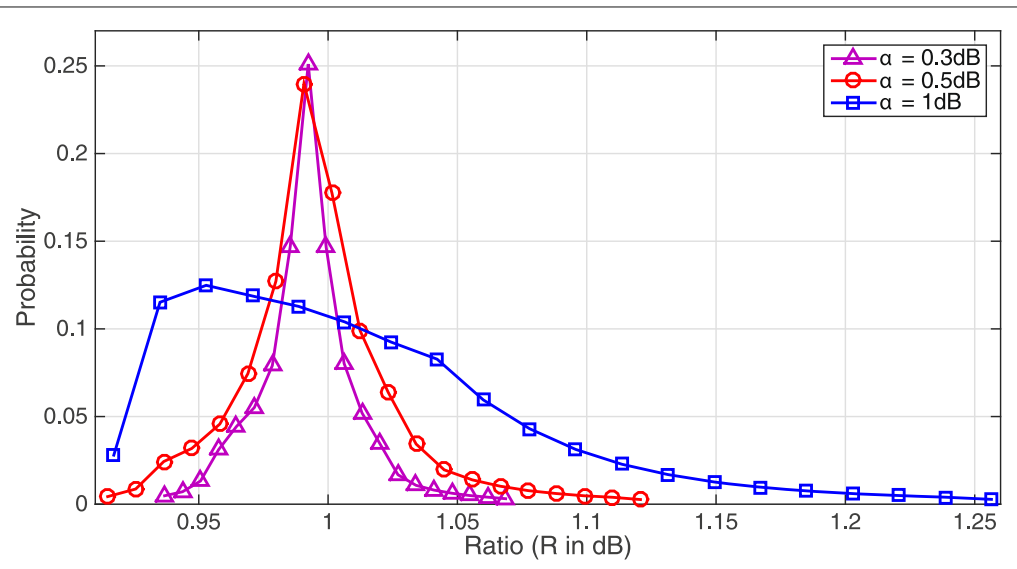

Fig. 5 The histogram of the ratio $\mathcal{R}$ in (16) 
Table 1 Probability of the ratio $\mathcal{R}$ falls in a range

\begin{tabular}{llll}
\hline Range of $\mathcal{R}$ in (16) & $\alpha=0.3 \mathrm{~dB}$ & $\alpha=0.5 \mathrm{~dB}$ & $\alpha=1 \mathrm{~dB}$ \\
\hline$(0.97,1.03)$ & 0.8473 & 0.7465 & 0.3570 \\
$(0.95,1.05)$ & 0.9713 & 0.8897 & 0.5884 \\
$(0.93,1.07)$ & 0.9992 & 0.9615 & 0.7913 \\
\hline
\end{tabular}

$\mathrm{dB}$ has a maximum stopband PSD performance of approximately $4 \mathrm{~dB}$-larger than that of the one with $\gamma=1.2$ $\mathrm{dB}$, and it still outperforms other waveforms in suppressing maximum stopband PSD. In Fig. 7, the normalized TBWs of the proposed, pure filtering, and pure windowing waveforms are chosen as $\eta=3.5$ so that less $\mathrm{VCs}$ are required. The proposed waveform using different SNR losses also outperforms other waveforms in suppressing maximum stopband PSD. Although WOLA has good stopband suppression performance for frequencies far from the passband, it will cause larger PSD at some frequencies near the stopband. Therefore, WOLA using R-C window will require more VCs to avoid possible ICI from other subbands compared to the proposed waveform.

\subsection{Bandwidth efficiency}

The proposed joint W-F waveform is more spectrally efficient than the other waveforms due to its capability of suppressing OOSBE to a specific level with a narrower guard band. As depicted in Fig. 3, the TBW is treated as the guard band between two adjacent subbands and is shared by two different users that are allocated to these subbands separately. Accordingly, the bandwidth efficiency is defined as

$$
\mathcal{E}(\eta)=\frac{P}{P+\eta} .
$$

Note that $\eta$ denotes the normalized TBW over the bandwidth of a subcarrier, and $P$ denotes the number of subcarriers in a subband.

The bandwidth efficiency performance of the various waveforms is listed in Table 2. These waveforms possess similar BER performances, which will be shown later. It is observed that the proposed waveform outperforms all the other waveforms at the price of approximately $0.5 \mathrm{~dB}$ SNR loss. Moreover, the proposed and the pure filtering waveforms can obtain better performances by relaxing the passband ripple constraint. These observations not only show the superiority of the proposed waveform in suppressing OOSBE spectral-efficiently but also suggest that the proposed optimization method offers more flexibility in designing the transmission waveform.

\subsection{Single-user BER simulations}

BER is considered as a metric for the performance of data transmission and reception in our simulations. QPSK or 16QAM without channel coding [42] is adopted as the modulation scheme with gray-coded symbols. Here, the simulations are executed based on the following cases.

(a) ISI-free case: The CP length is sufficient to eliminate ISI, and the transmitter is perfectly synchronized with the receiver. In this case, the proposed waveform,

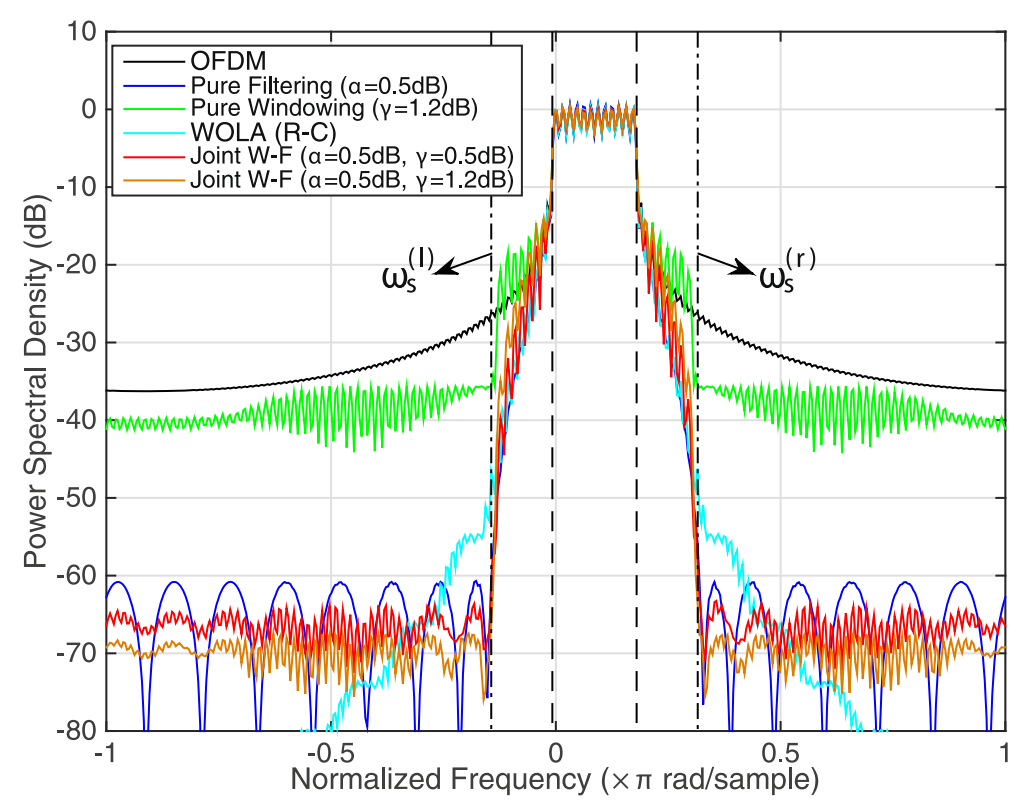

Fig. 6 PSD of various types of waveforms $(\eta=8.5)$ 


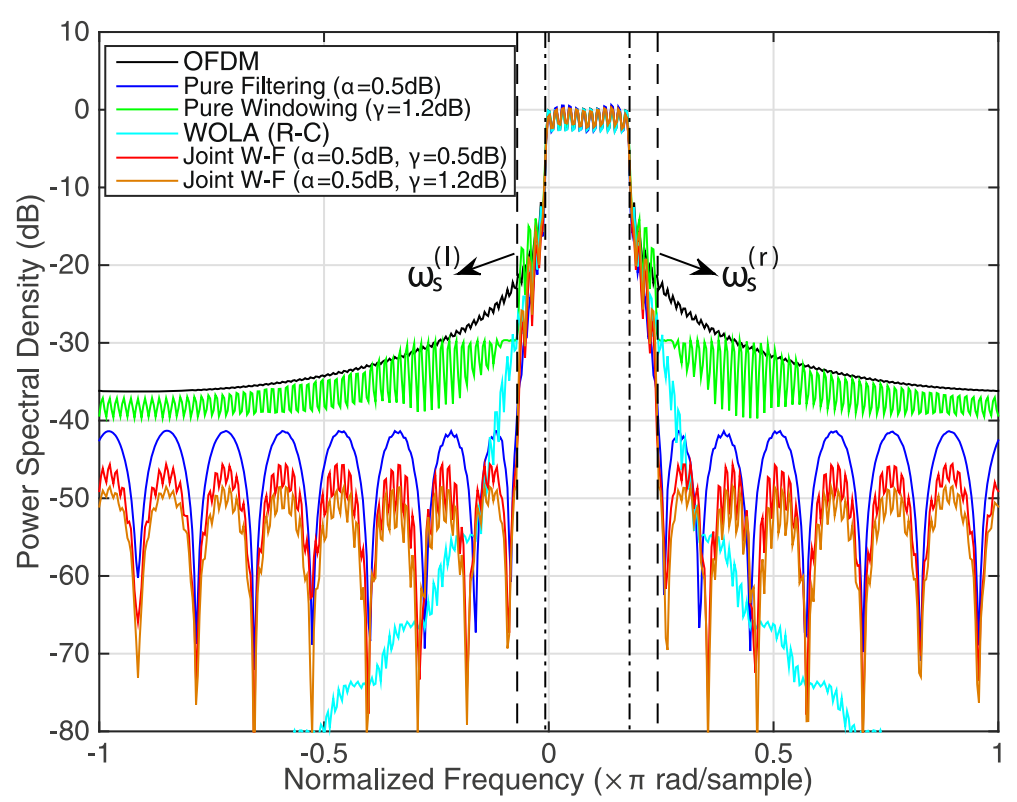

Fig. 7 PSD of various types of waveforms ( $\eta=3.5$ )

compared to OFDM, WOLA, and the pure filtering waveform, has a $0.5 \mathrm{~dB}$ BER disadvantage, which is roughly equal to the SNR loss. This phenomenon suggests that the proposed waveform trades a slight SNR loss for the advantageous bandwidth efficiency as shown in Table 2.

(b) ISI-incurring case: The CP length is insufficient so that there is ISI incurred at the receiver, and the transmitter is perfectly synchronized with the receiver. In this case, the proposed waveform is less prone to ISI compared to the pure filtering waveform (and WOLA) because it requires a shorter filter to achieve the same OOSBE suppression level.

Therefore, the proposed waveform can use a shorter CP to mitigate ISI, which makes it more spectrally efficient than the pure filtering waveform and WOLA.

The results of the ISI-free case are shown in Figs. 8 and 9. In the simulations, the normalized TBW is set as $\eta=3.5$. It is observed that the pure filtering waveform and

Table 2 Bandwidth efficiency comparison

\begin{tabular}{lllll}
\hline $\begin{array}{l}\text { Maximum } \\
\text { Stopband }\end{array}$ & WOLA & Pure Win. & Pure Fil. & Joint W-F \\
PSD (dB) & & $\gamma=1.2 \mathrm{~dB}$ & $\alpha: 0.5 / 1 \mathrm{~dB}$ & $\begin{array}{l}\alpha: 0.5 / 1 \mathrm{~dB} \\
\gamma=0.5 \mathrm{~dB}\end{array}$ \\
\hline-30 & $74 \%$ & $79 \%$ & $85.7 \% / 90.9 \%$ & $88.9 \% / 94.5 \%$ \\
-40 & $64.2 \%$ & $58.5 \%$ & $77.4 \% / 79 \%$ & $81.6 \% / 82.8 \%$ \\
-50 & $59.4 \%$ & $<50 \%$ & $67.8 \% / 68.6 \%$ & $71.9 \% / 75 \%$ \\
-60 & $<50 \%$ & $<50 \%$ & $59.4 \% / 60.9 \%$ & $64.2 \% / 65.9 \%$ \\
\hline
\end{tabular}

WOLA have BER performances similar to that of OFDM. The pure windowing waveform has an exact $1.2 \mathrm{~dB}$ disadvantage. Although the proposed waveform has an approximately $0.5 \mathrm{~dB}$ disadvantage (approximately equal to the SNR loss), it possesses the best OOSBE suppression performance as shown in Figs. 6 and 7. This advantage will be further demonstrated in Section 5.8.

In Figs. 10 and 11, the results of the ISI-incurring case are demonstrated. Here, the CP length is set as $L=23$. The filter lengths of the pure filtering and the proposed waveforms are set as $L_{f}=L=23$ and $L_{f}=17$, respectively. They are chosen this way so that the two waveforms possess a similar maximum stopband PSD of approximately $-44 \mathrm{~dB}$. The CS length of WOLA is $v=17$, and the resulting OOSBE suppression performance is $-26.56 \mathrm{~dB}$, and that of the pure windowing waveform with $1.2 \mathrm{~dB}$ SNR loss is $-29.83 \mathrm{~dB}$. In both plots, one can observe that the proposed waveform outperforms the pure filtering waveform and WOLA in the presence of ISI at the receiver. Although the BER performance of the pure windowing waveform is not be affected by ISI, its performance in suppressing OOSBE is poor, as demonstrated in Figs. 6 and 7. These results not only suggest that the proposed waveform requires a shorter filter length than the previous filtering-based waveform to achieve similar OOSBE suppression performances, but also indicate that the proposed waveform is less prone to ISI while possessing good capability of suppressing OOSBE compared to WOLA and the pure filtering waveform. 


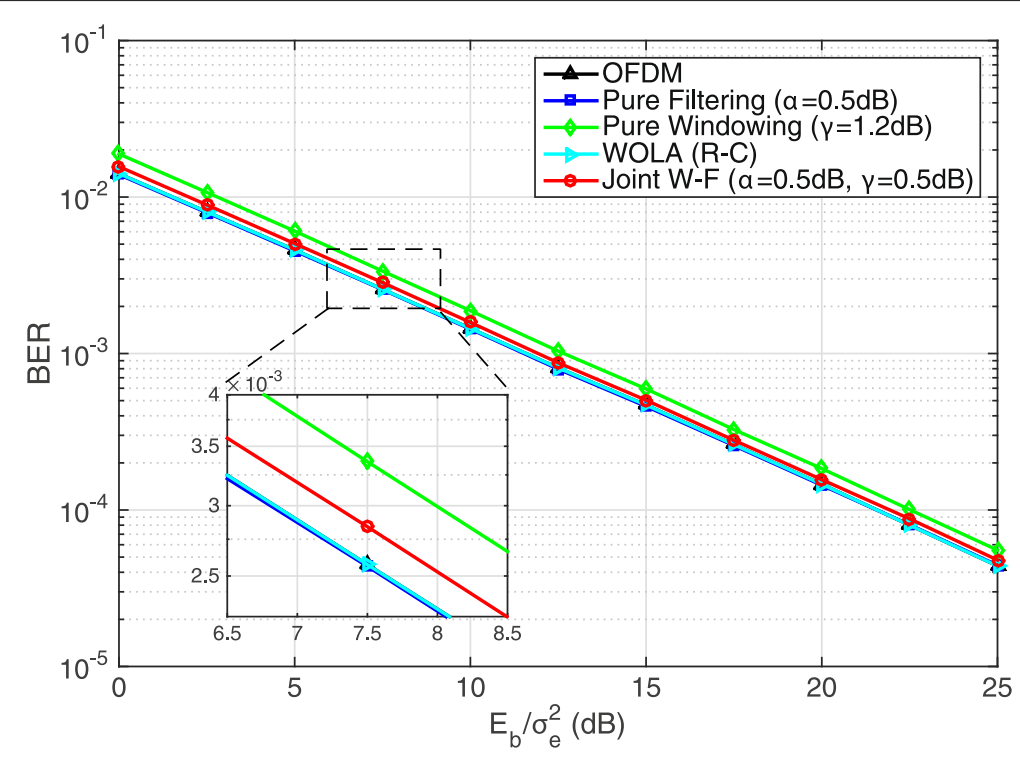

Fig. 8 ISI-free BER performances (QPSK)

\subsection{Asynchronous multi-user BER simulations}

Here, BER simulations are performed based on an asynchronous uplink scenario with multiple users modulated by the same waveform. The proposed waveform has the best performance especially in this scenario.

As suggested in [1-3], the requirement of relaxed frequency synchronization is based on the necessity of deploying a waveform with reduced OOSBE compared to conventional OFDM. Therefore, frequency asynchronism caused by carrier frequency offsets (CFOs) is introduced in our simulations. In [2], it was pointed out that the CFO of a user can be estimated and compensated at the receiver without requiring any information feedback from the user. Some example methods of estimating CFOs can be found in $[43,44]$. However, ICI will still occur because various CFOs of users are unable to be compensated for at the same time.

Suppose there are three users transmitting data symbols simultaneously, as illustrated in Fig. 3. The CFO of the user allocated to subband $\mathcal{K}$ is assumed to be perfectly estimated and compensated for at the receiver, while the other two users are allocated to neighboring subbands

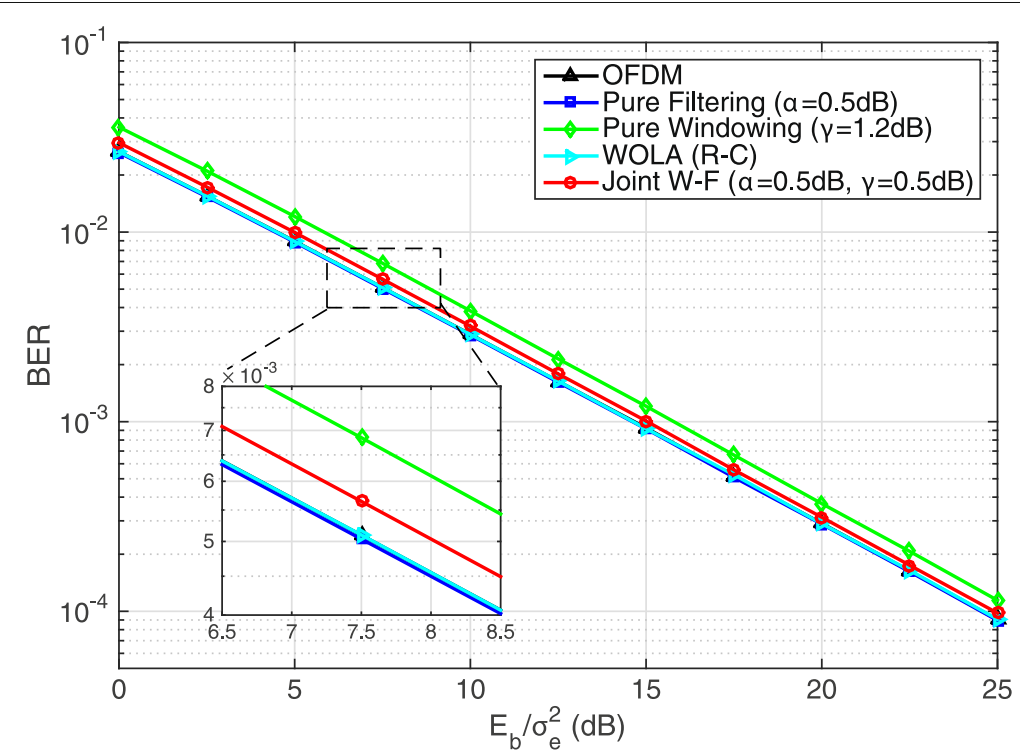

Fig. 9 ISI-free BER performances (16QAM) 


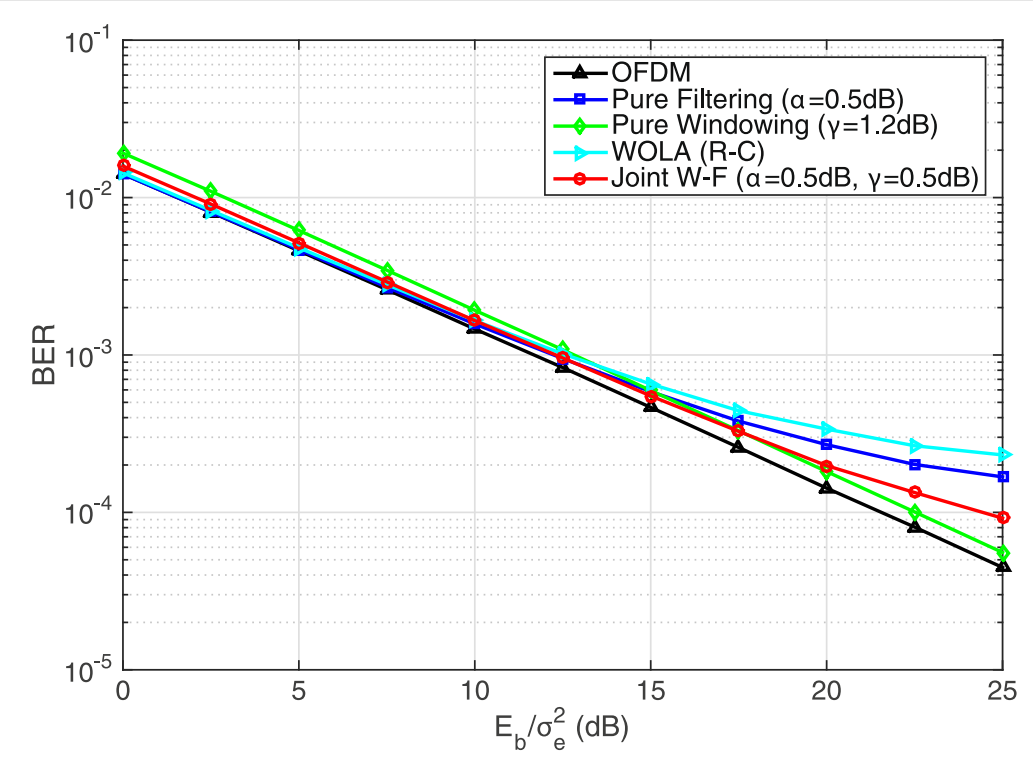

Fig. 10 ISI-incurring BER performances (QPSK)

and are asynchronous to the receiver, i.e., the CFOs of the neighboring users are not compensated for. Specifically, the two neighboring users, indexed user 1 and user 2, are allocated to subbands $\mathcal{K}_{1} \triangleq\{-P-\eta,-P-\eta-$ $1, \cdots,-1-\eta\}$ and $\mathcal{K}_{2} \triangleq\{P+\eta, P+\eta+1, \cdots, 2 P+$ $\eta-1\}$, respectively. The data symbols of the two users are denoted as $\mathbf{s}_{P}^{(1)}[n]$, and $\mathbf{s}_{P}^{(2)}[n]$, respectively. The precoded data symbols of user $l$ before adding $C P$ are denoted as $\mathbf{u}_{M}^{(l)}[n]=\mathbf{W}_{M}^{H} \mathbf{E}^{T}\left(\mathcal{K}_{l}\right) \mathbf{s}_{P}^{(l)}[n], l=1,2$. The window coefficients deployed by each user are the same; the filter functions used by user 1 and user 2 , denoted $f^{(1)}[n]$ and $f^{(2)}[n]$, respectively, are the frequency-shifted versions of the optimal filter function to the center frequencies of the allocated subbands, i.e.,

$$
\begin{aligned}
f^{(1)}[n] & =f[n] e^{-j \frac{2 \pi}{M}(P+\eta) n}, n=0,1, \cdots, L_{f}-1 \\
\text { and } f^{(2)}[n] & =f[n] e^{j \frac{2 \pi}{M}(P+\eta) n}, n=0,1, \cdots, L_{f}-1 .
\end{aligned}
$$

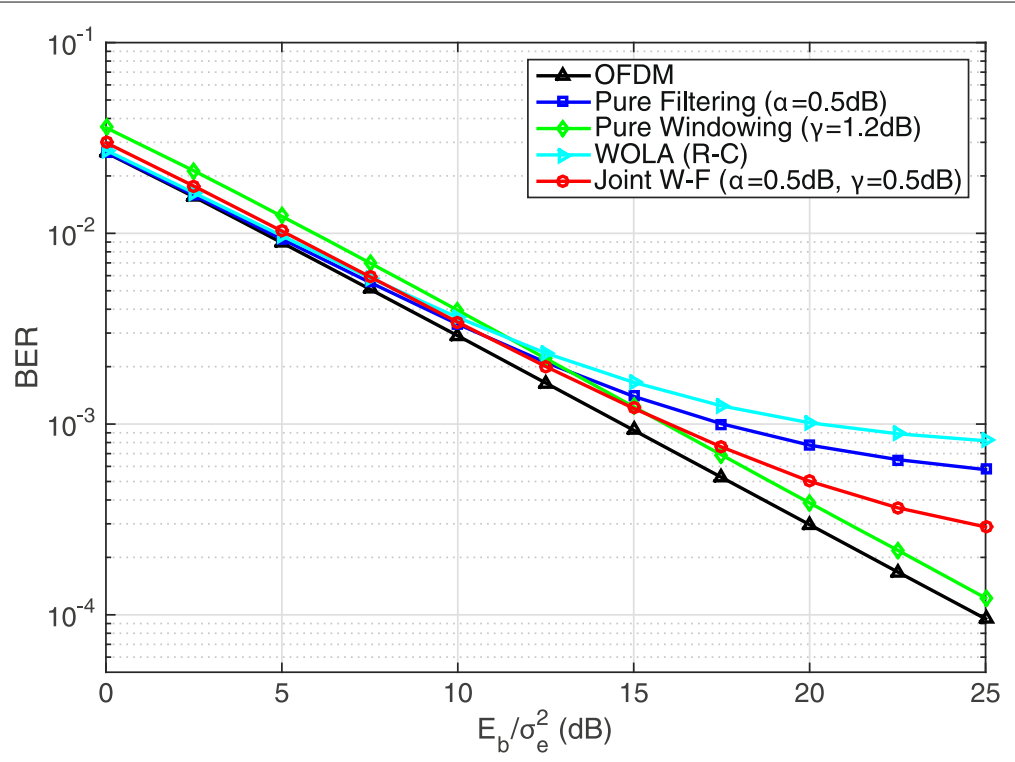

Fig. 11 ISI-incurring BER performances (16QAM) 


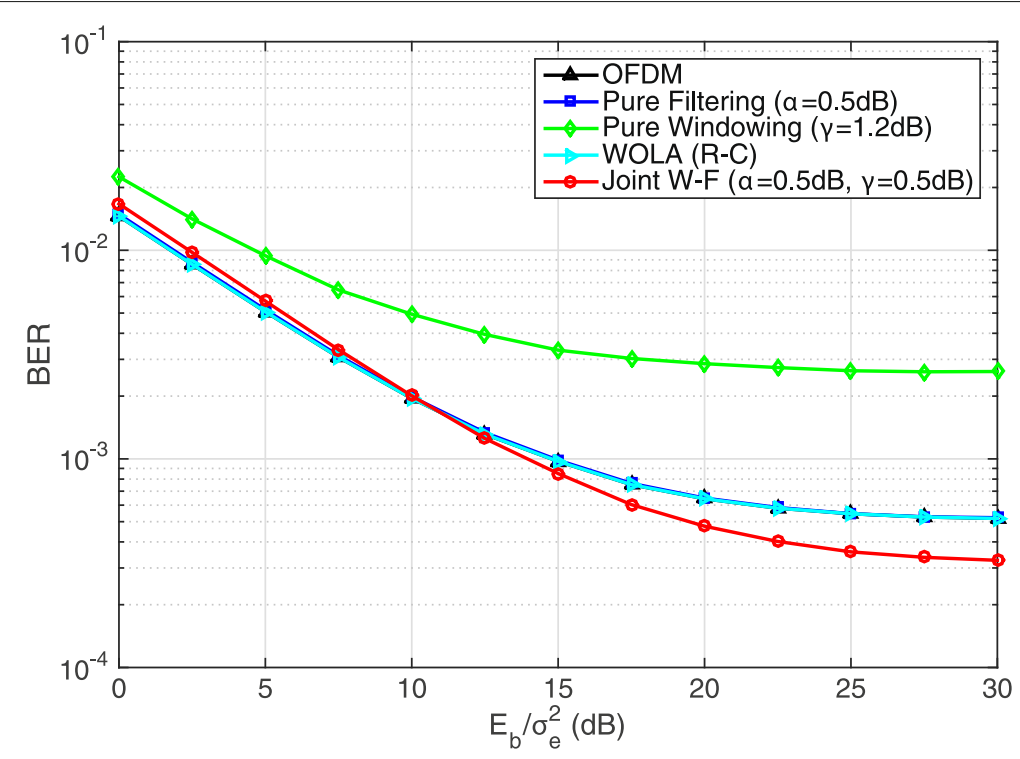

Fig. 12 Multi-user BER performances with CFOs (QPSK)

Let $h_{l}[n] \sim C N(0,1), n=0,1, \cdots, L_{h}$ denote the channel impulse response of user $l$, then the resulting effective channel is $c_{l}[n]=h_{l}[n] * f^{(l)}[n]$ where $*$ denotes the operation of convolution. For simplicity, the CP length is assumed to be sufficient to eliminate ISI. In the presence of CFO, the received signal vector after serial-to-parallel conversion and $\mathrm{CP}$ removal is expressed as

$$
\mathbf{y}_{M}[n]=\mathbf{C D u}_{M}[n]+\sum_{l=1}^{2} \Phi\left(\varepsilon_{l}\right) \mathbf{C}_{l} \mathbf{D} \mathbf{u}_{M}^{(l)}[n]+\mathbf{e}_{M}[n]
$$

where $\Phi\left(\varepsilon_{l}\right) \triangleq \operatorname{diag}\left(\left[1 e^{j \frac{2 \pi}{M} \varepsilon_{l}} \cdots e^{j \frac{2 \pi}{M}(M-1) \varepsilon_{l}}\right]\right)$ is the matrix representing phase shift incurred by the CFO, $\varepsilon_{l}$ denotes the normalized CFO of user $l$, and $\mathbf{C}_{l}$ is the circulant matrix consisting the effective channel's impulse response of user $l$ [44]. The decoding of $\mathbf{s}_{P}[n]$ is the same as performed in (10) and (11).

In Figs. 12 and 13, the BER simulation results of the user allocated to subband $\mathcal{K}$ are demonstrated. Here, the normalized CFOs are expressed as i.i.d. uniformly distributed random variables in the range of $[-\phi, \phi]$, as suggested in [44], where $\phi=0.1$. The normalized TBW is $\eta=1$.

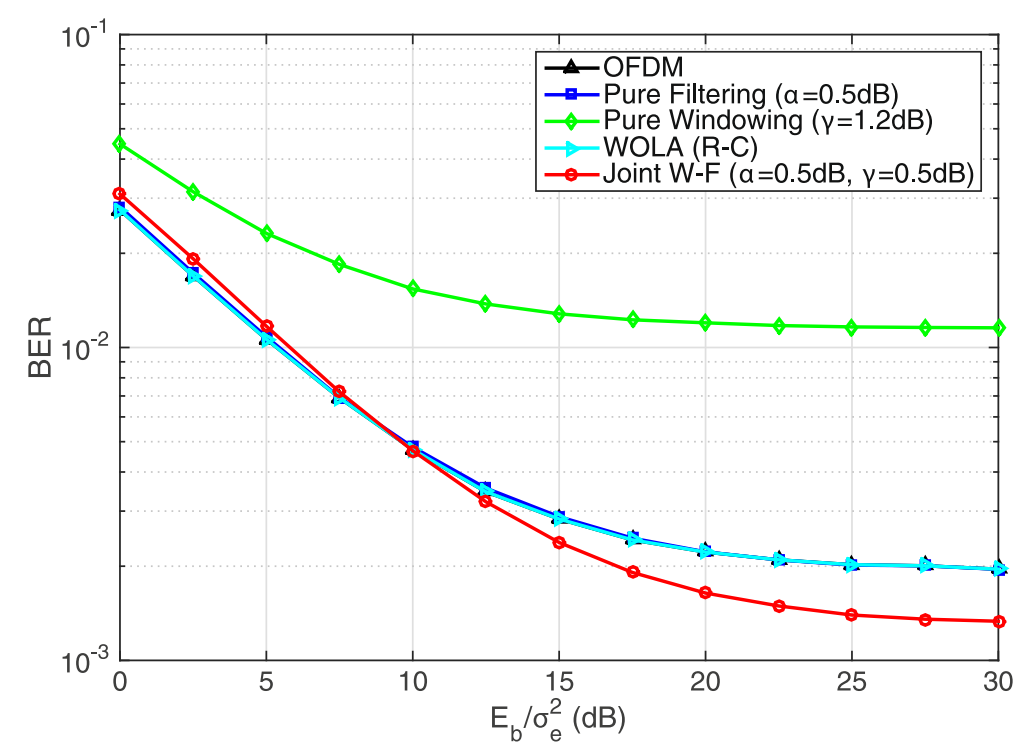

Fig. 13 Multi-user BER performances with CFOs (16QAM) 


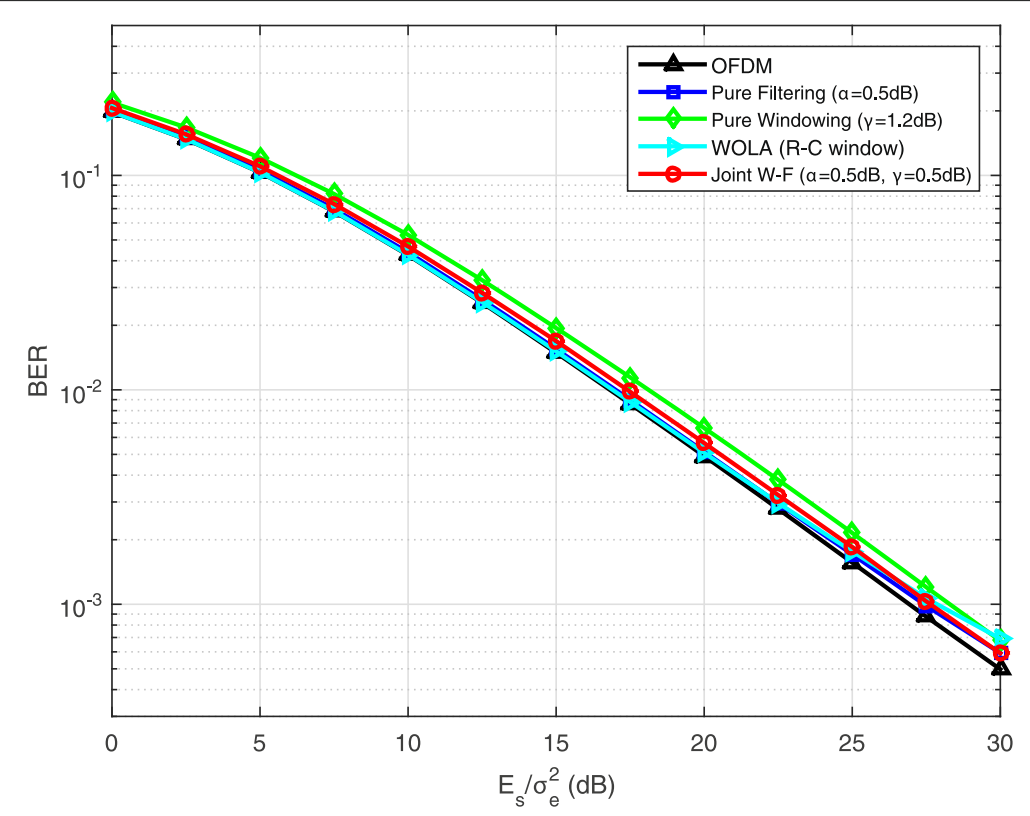

Fig. 14 BER performance with ETU channel model (16QAM, $L=16)$

The other parameters are set as presented in Section 5.1. The maximum stopband PSD of the proposed waveform (SNR loss $\gamma=0.5 \mathrm{~dB}$ ), the pure filtering waveform, and the pure windowing waveform are $-28.67 \mathrm{~dB},-24.51 \mathrm{~dB}$, and $-20.87 \mathrm{~dB}$, respectively. One can observe that the proposed waveform outperforms other waveforms significantly when $E_{b} / \sigma_{e}^{2}$ is larger than $12.5 \mathrm{~dB}$. This is because the proposed waveform has the best capability of suppressing OOSBE so that ICI introduced by CFOs can be greatly mitigated. Due to rectangular windowing and inferior capabilities in OOSBE suppression, the BER performances of OFDM, WOLA, and pure filtering waveform are similar. The pure windowing waveform has the worst BER performance since it will cause severe ICI due to destroying the orthogonality between subcarriers. In regimes of lower transmitting energy, i.e.,

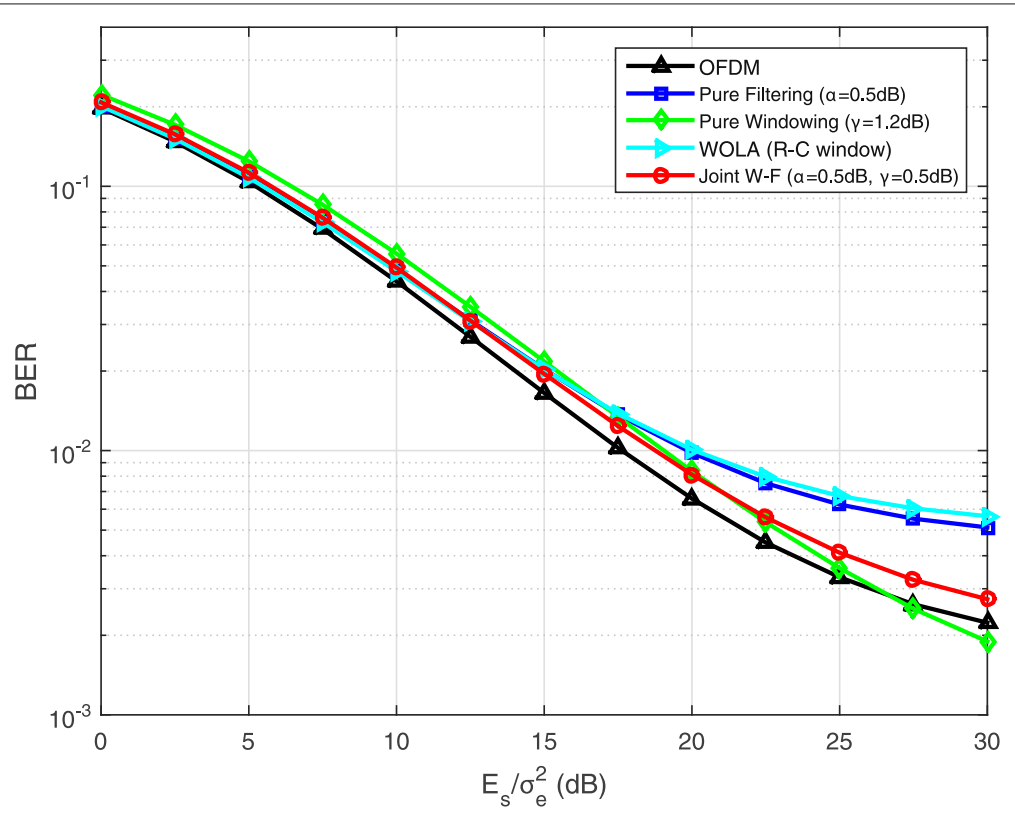

Fig. 15 BER performance with ETU channel model (16QAM, $L=8)$ 
Table 3 Comparison of the waveforms

\begin{tabular}{llllll}
\hline & OFDM & Pure Win. & Pure Fil. & WOLA & Joint W-F \\
\hline OOSBE & Large & Medium & Small & Small & Very small \\
SNR loss & None & Large & None & None & Small \\
CP extention for & None & None & Long & Long & Short \\
ISI mitigation & & & & & \\
\# of VCs for & Very & Large & Small & Small & Very small \\
ICI mitigation & Large & & & & \\
\hline
\end{tabular}

$E_{b} / \sigma_{e}^{2} \leq 12.5 \mathrm{~dB}$, the proposed waveform has a slight performance disadvantage compared to WOLA, the pure filtering waveform, and the conventional OFDM. This is because the BER performance of the zero-forcing receiver is more prone to noise rather than ICI in such regimes. These results suggest that the proposed waveform is more capable of suppressing ICI for asynchronous transmissions than other waveforms.

\subsection{BER simulations with ETU channel model}

BER simulations are executed based on the extended typical urban (ETU) channel model [45]. The results are demonstrated in Figs. 14 and 15. In these figures, the sampling frequency is $1.92 \mathrm{MHz}$, the velocity of the UE is $3 \mathrm{~km} / \mathrm{h}$, the number of subcarriers is $M=128$, and $\eta=1$. The filter length of the proposed joint W-F method is $L_{f}=9$, and $v=L_{f}$ for WOLA in both figures. In Fig. 14, the CP lengths of all the waveforms are set as $L=16$, and the filter length of the pure filtering one is $L_{f}=9$. One can observe that the joint W-F method also has a $0.5 \mathrm{~dB}$ performance disadvantage comparing with OFDM, WOLA, and the pure filtering method, which is the same as what was demonstrated in Fig. 9. In Fig. 15, the CP lengths of all the waveforms are set as $L=8$, and the filter length of the pure filtering one is $L_{f}=9$. The filter lengths are chosen so that the pure filtering and the proposed waveforms possess a similar maximum stopband PSD of approximately $-31 \mathrm{~dB}$. In this figure, it is also observed that the proposed waveform outperforms the pure filtering waveform and WOLA in the presence of ISI at the receiver, as demonstrated in Fig. 11.

\section{Conclusions}

In this paper, a joint windowing and filtering multi-carrier waveform was proposed. An iterative method for joint optimization was also proposed to minimize the OOSBE with controllable SNR loss at the receiver. In contrast with the previous pure filtering and pure windowing waveforms, the proposed waveform has an advantage in suppressing OOSBE to a lower level with similar BER performances. This advantage indicates that the proposed waveform is more spectrally efficient when suppressing OOSBE than the other waveforms. Moreover, the proposed waveform requires a shorter filter length and induces less SNR loss for achieving an OOSBE suppression specification compared to previous pure filtering and pure windowing waveforms, respectively. The detailed comparison of the proposed waveform with OFDM, the pure filtering waveform, the pure windowing waveform, and WOLA is presented in Table 3. Simulation results support the above advantages of the proposed waveform.

Channel estimation and peak-to-average power ratio (PAPR) reduction remain as open problems. Extending the proposed waveform to a multiple-input-multipleoutput system is also of interest.

\section{Appendix 1: Derivation of constraint (27c)}

By substituting (24) into (4), the PSD of the transmitted signal $x[n]$ is expressed as

$$
\mathcal{S}_{x}\left(e^{j \omega}\right)=\frac{E_{s}}{N} \mathbf{w}^{H} \mathbf{R}^{T} \mathbf{V}\left(e^{j \omega}\right) \mathbf{R} \mathbf{w} \mathbf{a}^{T}\left(e^{j \omega}\right) \mathbf{r}_{f}
$$

where $\mathbf{V}\left(e^{j \omega}\right)=\sum_{k \in \mathcal{K}} \mathbf{v}_{N}\left(e^{j\left(\omega-\Omega_{k}\right)}\right) \mathbf{v}_{N}^{H}\left(e^{j\left(\omega-\Omega_{k}\right)}\right)$. By integrating (37) from $\pi$ to $-\pi$,

$$
\int_{-\pi}^{\pi} \mathcal{S}_{x}\left(e^{j \omega}\right) \frac{\mathrm{d} \omega}{2 \pi}=\frac{E_{s}}{N} \underbrace{\mathbf{w}^{H} \mathbf{R}^{T} \int_{-\pi}^{\pi} \mathbf{V}\left(e^{j \omega}\right) \mathbf{R} \mathbf{w} \mathbf{a}^{T}\left(e^{j \omega}\right) \frac{\mathrm{d} \omega}{2 \pi}}_{\mathbf{q}^{T}} \mathbf{r}_{f} .
$$

Next, denote the $i$ th entry of vector $\mathbf{a}\left(e^{j \omega}\right)$ as $\left[\mathbf{a}\left(e^{j \omega}\right)\right]_{i}=$ $e^{j\left(L_{f}-i\right) \omega}$. Then, the $i$ th entry of vector $\mathbf{q}$ is written as

$$
[\mathbf{q}]_{i}=\mathbf{w}^{H} \mathbf{R}^{T} \underbrace{\int_{-\pi}^{\pi} \mathbf{V}\left(e^{j \omega}\right) e^{j\left(L_{f}-i\right) \omega} \frac{\mathrm{d} \omega}{2 \pi}}_{\mathbf{Q}_{i}} \mathbf{R} \mathbf{w} .
$$

Let the $(m, n)$ th entry of matrix $\mathbf{V}\left(e^{j \omega}\right)$ be $\left[\mathbf{V}\left(e^{j \omega}\right)\right]_{m, n}=\sum_{k \in \mathcal{K}} e^{j(m-n)\left(\omega-\Omega_{k}\right)}$. Then, the $N \times N$ matrix $\mathbf{Q}_{i}$ is given by

$$
\begin{aligned}
{\left[\mathbf{Q}_{i}\right]_{m, n} } & =\sum_{k \in \mathcal{K}} e^{j(n-m) \Omega_{k}} \int_{-\pi}^{\pi} e^{j\left(m-n+L_{f}-i\right) \omega} \frac{\mathrm{d} \omega}{2 \pi} \\
& = \begin{cases}\sum_{k \in \mathcal{K}} e^{j(n-m) \Omega_{k}}, & m-n+L_{f}-i=0 \\
0, & \text { else. }\end{cases}
\end{aligned}
$$

By substituting Eq. (38) into (5), constraint (27c) is obtained.

\section{Appendix 2: Proof of Theorem 1}

(a) First, prove that $\mathrm{D}(\boldsymbol{\theta})$ is a convex set. Let $\hat{\mathbf{w}}_{1}=\left(\mathbf{1}_{2} \otimes \boldsymbol{\tau}\right) \circ \mathbf{d}(\boldsymbol{\theta})$ and $\hat{\mathbf{w}}_{2}=\left(\mathbf{1}_{2} \otimes \boldsymbol{\tau}^{\prime}\right) \circ \mathbf{d}(\boldsymbol{\theta})$ be two vectors in $D(\boldsymbol{\theta})$, then

$$
\lambda \hat{\mathbf{w}}_{1}+(1-\lambda) \hat{\mathbf{w}}_{2}=\left\{\mathbf{1}_{2} \otimes\left[\lambda \boldsymbol{\tau}+(1-\lambda) \boldsymbol{\tau}^{\prime}\right]\right\} \circ \mathbf{d}(\boldsymbol{\theta}) \in \mathrm{D}(\boldsymbol{\theta})
$$

for all $m=1, \cdots, M$ and $\lambda \in[0,1]$. Therefore, $\mathrm{D}(\boldsymbol{\theta})$ is a convex set. 
Followed by the argument of dom $\hat{\sigma}$, define

$$
\begin{aligned}
g(\boldsymbol{\tau}) & =\left.\hat{\sigma}(\hat{\mathbf{w}})\right|_{\hat{\mathbf{w}}=\left(\mathbf{1}_{2} \otimes \boldsymbol{\tau}\right) \circ \mathbf{d}(\boldsymbol{\theta})} \\
& =\frac{1}{M} \sum_{m=1}^{M} \frac{1}{\tau_{m}^{2}}, \operatorname{dom} g=\mathrm{R}_{++}^{M},
\end{aligned}
$$

where $\tau_{m} \triangleq[\boldsymbol{\tau}]_{m}$. This definition implies that $\hat{\sigma}$ is convex if $g$ is convex. The convexity of $g$ is shown by its second-order condition [30]. By taking the second-order derivatives of $g$, the Hessian is written as

$$
\nabla^{2} g(\boldsymbol{\tau})=\frac{6}{M} \operatorname{diag}\left(\left[\begin{array}{llll}
\tau_{1}^{-4} & \tau_{2}^{-4} & \cdots & \tau_{M}^{-4}
\end{array}\right]\right),
$$

which is a positive definite matrix. Therefore, $\hat{\sigma}(\hat{\mathbf{w}})$ is convex.

(b) By taking second-order derivatives of $\hat{\sigma}$, the Hessian is given by

$$
\left[\nabla^{2} \hat{\sigma}(\hat{\mathbf{w}})\right]_{m, n}= \begin{cases}\frac{8}{M} \hat{w}_{m}^{2} \hat{\sigma}_{m}^{3}-\frac{2}{M} \hat{\sigma}_{m}^{2}, & m=n \\ \frac{8}{M} \hat{w}_{m} \hat{w}_{n} \hat{\sigma}_{m}^{3}, & |m-n|=M \\ 0, & \text { else }\end{cases}
$$

where $\hat{\sigma}_{m}=1 /\left(\hat{w}_{m}^{2}+\hat{w}_{m+M}^{2}\right)$ for $1 \leq m \leq M$, and $\hat{\sigma}_{m}=1 /\left(\hat{w}_{m}^{2}+\hat{w}_{m-M}^{2}\right)$ for $M+1 \leq m \leq 2 M$. The Hessian matrix $\nabla^{2} \hat{\sigma}(\hat{\mathbf{w}})$ is not positive semidefinite. This property can be shown by considering the following example: $\hat{\mathbf{w}}=\frac{1}{\sqrt{2}} \mathbf{1}_{2 M}$. The resulting minimum eigenvalue of the Hessian is $-2 / M$. Therefore, the function $\hat{\sigma}$ is nonconvex.

\section{Abbreviations}

CFO: Carrier frequency offset; CP: Cyclic prefix; CS: Cyclic suffix; DFT: Discrete fourier transform; f-OFDM: Filtered-OFDM; FBMC: Filter bank multi-carrier; FFT: Fast fourier transform; FIR: Finite impulse response; GFDM: Generalized frequency division multiplexing; ICl: Inter-carrier interference; IFFT: Inverse FFT; IPM: Interior-point method; ISI: Inter-symbol interference; OFDM: Orthogonal frequency division multiplexing; OOSBE: Out-of subband emissions; PAPR: Peak-to-average power ratio; PSD: Power spectral density; R-C: Raised-cosine; SNR: Signal-to-noise ratio; TBW: Transition bandwidth; UFMC: Universal-filtered multi-carrier; VC: Virtual carrier; WOLA: Weighted overlap-and-add

\section{Acknowledgements}

The authors would like to thank Dr. Gordon L. Stüber, Joseph M. Pettit Chair Professor of ECE department, Georgia Institute of Technology, for his valuable comments that significantly improved the quality of this paper.

\section{Funding}

This work is supported by the Ministry of Science and Technology, Taiwan, under contracts MOST 106-2221-E-002-034.

\section{Availability of data and materials}

Not available online. Please contact author for data requests.

\section{Authors' contributions}

MFT made the main contributions to the joint window and filter optimization algorithms' design and experiments, as well as drafting the manuscript. BCS checked the manuscript and offered critical suggestions to design the algorithm. Both authors read and approved the final manuscript.

\section{Competing interests}

The authors declare that they have no competing interests.

\section{Publisher's Note}

Springer Nature remains neutral with regard to jurisdictional claims in published maps and institutional affiliations.

\begin{abstract}
Author details
${ }^{1}$ Graduate Institute of Communication Engineering, National Taiwan University, No.1, Sec. 4, Roosevelt Road, Taipei 10617, Taiwan. ${ }^{2}$ Department of Electrical Engineering, National Taiwan University, No.1, Sec. 4, Roosevelt Road, Taipei 10617, Taiwan.
\end{abstract}

Received: 6 February 2018 Accepted: 29 August 2018

Published online: 03 October 2018

\section{References}

1. J. G. Andrews, S. Buzzi, W. Choi, S. V. Hanly, A. Lozano, A. C. K. Soong, J. C. Zhang, What will 5 G be?. IEEE J. Sel. Areas Commun. 32(6), 1065-1082 (2014)

2. G. Wunder, P. Jung, M. Kasparick, T. Wild, F. Schaich, Y. Chen, S. T. Brink, I. Gaspar, N. Michailow, A. Festag, L. Mendes, N. Cassiau, D. Ktenas, M. Dryjanski, S. Pietrzyk, B. Eged, P. Vago, F. Wiedmann, 5GNOW: non-orthogonal, asynchronous waveforms for future mobile applications. IEEE Commun. Mag. 52(2), 97-105 (2014)

3. X. Zhang, L. Chen, J. Qiu, J. Abdoli, On the waveform for 5G. IEEE Commun. Mag. 54(11), 74-80 (2016)

4. A. A. Zaidi, R. Baldemair, H. Tullberg, H. Bjorkegren, L. Sundstrom, J. Medbo, C. Kilinc, I. D. Silva, Waveform and numerology to support $5 \mathrm{G}$ services and requirements. IEEE Commun. Mag. 54(11), 90-98 (2016)

5. L. Zhang, A. Ijaz, P. Xiao, A. Quddus, R. Tafazolli, Subband filtered multi-carrier systems for multi-service wireless communications. IEEE Trans. Wirel. Commun. 16(3), 1893-1907 (2017)

6. L. Zhang, A. ljaz, P. Xiao, R. Tafazolli, Multi-service system: an enabler of flexible 5G air interface. IEEE Commun. Mag. 55(10), 152-159 (2017)

7. P. Guan, D. Wu, T. Tian, J. Zhou, X. Zhang, L. Gu, A. Benjebbour, M. Iwabuchi, Y. Kishiyama, $5 \mathrm{G}$ field trials: OFDM-based waveforms and mixed numerologies. IEEE J. Sel. Areas Commun. 35(6), 1234-1243 (2017)

8. B. Farhang-Boroujeny, OFDM versus filter bank multicarrier. IEEE Signal Proc. Mag. 28(3), 92-112 (2011)

9. X. Mestre, M. Majoral, S. Pfletschinger, An asymptotic approach to parallel equalization of filter bank based multicarrier signals. IEEE Trans. Signal Process. 61(14), 3592-3606 (2013)

10. M. Pauli, P. Kuchenbecker, in Proc. IEEE Int. Conf. Commun., vol. 3. On the reduction of the out-of-band radiation of OFDM-signals (IEEE, Atlanta, 1998), pp. 1304-1308

11. Y. Medjahdi, R. Zayani, H. Shaïk, D. Roviras, in 2017 IEEE International Conference on Communications Workshops ICC Workshops). WOLA processing: a useful tool for windowed waveforms in $5 \mathrm{G}$ with relaxed synchronicity (IEEE, Paris, 2017), pp. 393-398

12. Y.-P. Lin, S. M. Phoong, Window designs for DFT-based multicarrier systems. IEEE Trans. Signal Process. 53(3), 1015-1024 (2005)

13. D. Roque, C. Siclet, Performances of weighted cyclic prefix OFDM with low-complexity equalization. IEEE Trans. Commun. 17(3), 439-442 (2013)

14. N. Michailow, M. Matthé, I. S. Gaspar, A. N. Caldevilla, L. L. Mendes, A. Festag, G. Fettweis, Generalized frequency division multiplexing for 5 th generation cellular networks. IEEE Trans. Commun. 62(9), 3045-3061 (2014)

15. H. M. Chen, W. C. Chen, C. D. Chung, Spectrally precoded OFDM and OFDMA with cyclic prefix and unconstrained guard ratios. IEEE Trans. Wirel. Commun. 10(5), 1416-1427 (2011)

16. M. Ma, X. Huang, B. Jiao, Y. J. Guo, Optimal orthogonal precoding for power leakage suppression in DFT-based systems. IEEE Trans. Commun. 59(3), 844-853 (2011)

17. A. Farhang, N. Marchetti, L. E. Doyle, Low-complexity modem design for GFDM. IEEE Trans. Signal Process. 64(6), 1507-1518 (2016)

18. V. Vakilian, T. Wild, F. Schaich, S. ten Brink, J. F. Frigon, in 2013 IEEE Globecom Workshops (GC Wkshps). Universal-filtered multi-carrier technique for wireless systems beyond LTE (IEEE, Atlanta, 2013), pp. 223-228

19. T. Wild, F. Schaich, Y. Chen, in 2014 19th International Conference on Digital Signal Processing. $5 \mathrm{G}$ air interface design based on universal filtered (UF-)OFDM (IEEE, Hong Kong, 2014), pp. 699-704

20. M.-F. Tang, B. Su, in 2016 IEEE International Conference on Communications Workshops (ICC). Filter optimization of low out-of-subband emission for universal-filtered multicarrier systems (IEEE, Kuala Lumpur, 2016), pp. 468-473

21. L. Zhang, P. Xiao, A. Quddus, Cyclic prefix-based universal filtered multicarrier system and performance analysis. IEEE Trans. Signal Process. 23(9), 1197-1201 (2016) 
22. J. Abdoli, M. Jia, J. Ma, in 2015 IEEE 16th International Workshop on Signal Processing Advances in Wireless Communications (SPAWC). Filtered OFDM: a new waveform for future wireless systems (IEEE, Stockholm, 2015), pp. 66-70

23. X. Zhang, M. Jia, L. Chen, J. Ma, J. Qiu, in 2015 IEEE Global Communications Conference (GLOBECOM). Filtered-ofdm - enabler for flexible waveform in the 5th generation cellular networks (IEEE, San Diego, 2015), pp. 1-6

24. P. Weitkemper, J. Bazzi, K. Kusume, A. Benjebbour, Y. Kishiyama, in 2016 IEEE International Conference on Communications Workshops (ICC). Adaptive filtered OFDM with regular resource grid (IEEE, Kuala Lumpur, 2016), pp. 462-467

25. D. J. Han, J. Moon, D. Kim, S. Y. Chung, Y. H. Lee, Combined subband-subcarrier spectral shaping in multi-carrier modulation under the excess frame length constraint. IEEE J. Sel. Areas Commun. 35(6), 1339-1352 (2017)

26. Y.-P. Lin, S.-M. Phoong, P. Vaidyanathan, Filter bank transceivers for OFDM and DMT systems. (Cambridge University Press, Cambridge, United Kingdom, 2010)

27. J. A. Zhang, X. Huang, A. Cantoni, Y. J. Guo, Sidelobe suppression with orthogonal projection for multicarrier systems. IEEE Trans. Commun. 60(2), 589-599 (2012)

28. P.P. Vaidyanathan, Multirate systems and filter banks. (Prentice-Hall, New Jersey, 1993)

29. K. Ichige, M. Iwaki, R. Ishii, Accurate estimation of minimum filter length for optimum FIR digital filters. IEEE Trans. Circ. Syst. II: Analog Digit. Signal Process. 47(10), 1008-1016 (2000)

30. S. Boyd, L. Vandenberghe, Convex optimization. (Cambridge university press, Cambridge, United Kingdom, 2004)

31. A. Papoulis, Signal analysis vol. 191. (McGraw-Hill, New York, 1977)

32. S.-P. Wu, S. Boyd, L. Vandenberghe, in Proceedings of 35th IEEE Conference on Decision and Control, vol. 1. FIR filter design via semidefinite programming and spectral factorization (IEEE, Kobe, 1996), pp. 271-276

33. A. S. Alkhairy, K. G. Christian, J. S. Lim, Design and characterization of optimal FIR filters with arbitrary phase. IEEE Trans Signal Process. 41(2), 559-572 (1993)

34. M. Grant, S. Boyd, CVX: MATLAB Software for disciplined convex programming, version 2.1 (2014). http://cvxr.com/cvx

35. R. G. Lorenz, S. P. Boyd, Robust minimum variance beamforming. IEEE Trans. Signal Process. 53(5), 1684-1696 (2005)

36. Z. L. Yu, W. Ser, M. H. Er, Z. Gu, Y. Li, Robust adaptive beamformers based on worst-case optimization and constraints on magnitude response. IEEE Trans. Signal Process. 57(7), 2615-2628 (2009)

37. S. W. Peters, R. W. Heath, in 2009 IEEE International Conference on Acoustics, Speech and Signal Processing. Interference alignment via alternating minimization (IEEE, Taipei, 2009), pp. 2445-2448

38. L. Kong, S. Han, C. Yang, Hybrid Precoding With Rate and Coverage Constraints for Wideband Massive MIMO Systems. IEEE Trans. Wirel. Commun. 17(7), 4634-4647 (2018)

39. M. Colombo, J. Gondzio, Further development of multiple centrality correctors for interior point methods. Comput. Optim. Appl. 41(3), 277-305 (2008)

40. T. Wild, F. Schaich, in 2015 IEEE 81st Vehicular Technology Conference (VTC Spring). A reduced complexity transmitter for UF-OFDM (IEEE, Glasgow, 2015), pp. 1-6

41. G. Cuypers, K. Vanbleu, G. Ysebaert, M. Moonen, P. Vandaele, in Communications, 2003. ICC'03. IEEE International Conference On, vol. 4. Combining raised cosine windowing and per tone equalization for RFI mitigation in DMT receivers (IEEE, Anchorage, 2003), pp. 2852-28564

42. J. G. Proakis, Digital communications, 4th edn. (McGraw-Hill, New York, 2001)

43. H. T. Hsieh, W. R. Wu, Blind maximum-likelihood carrier-frequency-offset estimation for interleaved OFDMA uplink systems. IEEE Trans. Veh. Technol. 60(1), 160-173 (2011)

44. X. N. Zeng, A. Ghrayeb, Joint CFO and channel estimation for OFDMA uplink: an application of the variable projection method. IEEE Trans. Wirel. Commun. 8(5), 2306-2311 (2009)

45. S. Sesia, M. Baker, I. Toufik, LTE - the UMTS long term evolution: from theory to practice. (John Wiley \& Sons, New Jersey, 2011)

\section{Submit your manuscript to a SpringerOpen ${ }^{\circ}$ journal and benefit from:}

\section{- Convenient online submission}

Rigorous peer review

- Open access: articles freely available online

- High visibility within the field

- Retaining the copyright to your article

Submit your next manuscript at $>$ springeropen.com 